This is an Accepted Manuscript of an article published by Springer Verlag in "Microfluidics and Nanofluidics" on $19^{\text {th }}$ March 2016, available online:

http://link.springer.com/article/10.1007\%2Fs10404-016-1718-1 


\title{
Hydrodynamic dispersion in long micro-channels under conditions of electroosmotic circulation. II. Electrolytes
}

\author{
E.E Licon Bernal ${ }^{\mathrm{a}^{*}}$, V.I.Kovalchuk ${ }^{\mathrm{b}}$, E.K.Zholkovskiy ${ }^{\mathrm{b}}$, A.Yaroshchuk $^{\mathrm{a}, \mathrm{c}}$ \\ ${ }^{a}$ Department of Chemical Engineering, Polytechnic University of Catalonia, Diagonal 647, 08028 \\ Barcelona, Spain \\ ${ }^{b}$ Institute of Bio-Colloid Chemistry, National Academy of Sciences of Ukraine, Vernadskogo 42, 03142 \\ Kiev, Ukraine \\ ' ICREA, Passeig Lluís Companys 23, 08010 Barcelona, Spain \\ *Corresponding author: Edxon Eduardo Licon Bernal \\ Email: edxon.eduardo.licon@upc.edu ; Phone:(+34) 934016997
}

\section{Abstract}

This work describes the steady state transport of an electrolyte due to a stationary concentration difference in straight long channels under conditions of electroosmotic circulation. The electroosmotic flow is induced due to the slip produced at the charged channel walls. This flow is assumed to be compensated by a pressure-driven counter-flow so that the net volume flow through the channel is exactly zero. Owing to the concentration dependence of electroosmotic slip there is an involved coupling between the solute transfer, hydrodynamic flow and charge conservation. Nevertheless, for such a system the Taylor-Aris Dispersion (TAD) theory is shown to be approximately applicable locally within an inner part of the channel for a wide range of Péclet numbers (Pe) irrespective of the concentration difference. Numerical simulations reveal only small deviations from analytical solutions for the inner part of the channel. The breakdown of TAD theory occurs within boundary regions near the channel ends and - is related to the variation of the dispersion mechanism from the purely molecular diffusion at the channel ends to the hydrodynamic dispersion within the inner part of the channel. This boundary region is larger at the lower-concentration channel edge and its size increases nearly linearly with $P e$ number. It is possible to derive a simple analytical approximation for the inner profile of cross-section-averaged electrolyte concentration in terms of only few parameters, determined numerically. Such analytical approximations can be useful for experimental studies of concentration-polarization phenomena in long micro-channels.

\section{Keywords}

Hydrodynamic dispersion, Taylor-Aris dispersion, convection-diffusion, straight long microchannel, electrolyte transport, electroosmotic circulation. 


\section{Introduction}

The phenomenon of concentration polarization $(\mathrm{CP})$ is caused by the difference in the transport numbers of ions between two adjacent media, for example, on the boundary between an ionexchange membrane and an electrolyte solution. Due to this difference, the electro-migration flow of an ion produced by an externally applied electric field is discontinuous at the interface. In order to ensure ion conservation, a gradient of concentration of this ion arises in the system close to the interface. The diffusion flux associated with this concentration gradient compensates the electro-migration flux discontinuity.

$\mathrm{CP}$ is a fundamental phenomenon which can manifest itself in electrode systems or in microheterogeneous systems, such as semipermeable membranes, ion-exchange resins, colloid dispersions, etc. (Newman 2004). It leads to concentration over-potentials, limiting-current effects, reduction of selectivity and productivity of membrane processes, etc. (Newman 2004; Strathmann 2002; Baker 2004). CP manifests itself also in the process of formation of LangmuirBlodgett films (LB-films) by the deposition of charged Langmuir monolayers on a substrate surface (Kovalchuk et al. 2011).

$\mathrm{CP}$ has been investigated experimentally in micro/nano fluidic systems, for example in some studies where considerable current-induced enrichment/depletion in the concentration of charged fluorescent markers was reported to have been directly observed close to micro/nanointerfaces (Kovarik et al. 2009; Zangle et al. 2009). Furthermore, nonlinear and time-dependent current-voltage characteristics have also been observed that can be interpreted in terms of concentration-polarization phenomena. (Kim et al. 2007; Kelly et al. 2009). This phenomenon as applied to micro/nano fluidic systems has also been considered theoretically in a number of recent publications (Postler et al. 2008; Zangle et al. 2010; Yaroshchuk et al. 2011).

Some specific manifestations of CP in microfluidic systems have been studied. For example, the concentration "shock waves" that occur in current-polarized sub-micro-channels in contact with nano-channels have been predicted for the first time by (Mani et al. 2009) and further explored in (Mani and Bazant 2011; Dydek et al. 2011; Yaroshchuk 2012; Deng et al. 2013). In another publication (Zangle et al. 2010), the authors considered the implications of depletion and enrichment "shocks" for the analyte pre-concentration via stacking and/or focusing. Several sample pre-concentration modes coupled to CP phenomena were also studied numerically by (Plecis et al. 2008) within the scope of 1D approach. (Strickland et al. 2010) and 
(Suss et al. 2011) studied the impact of CP phenomena on the operation of nano-porous electroosmotic pumps and found the $\mathrm{CP}$ propagation to be important for their performance. The existence of moving sharp concentration "fronts" has also been predicted theoretically for current-polarized joined nano-/micro-systems with strong hydrodynamic dispersion in their micro-channel parts (Yaroshchuk et al. 2011).

According to the previous study (Part I, Licon Bernal et al. 2014), the overlap of a pressure driven flow with electroosmotic flow (Electroosmotic circulation, EOC) in micro-channels can give rise to strong hydrodynamic dispersion and result in a peculiar distribution of solutes in them. It was demonstrated that under conditions of a steady zero net flow (zero cross-section averaged longitudinal velocity) the longitudinal profile of cross-section-averaged concentration of uncharged solute is linear and the Taylor-Aris Dispersion theory (TAD) (Taylor 1953; Aris 1956) is applicable within a major inner part of a long narrow channel. The applicability of the TAD to the description of mass transport in narrow long channels under conditions of EOC has been the subject of some recent studies (Yaroshchuk et al. 2011; Rubinstein and Zaltzman 2013). (Yaroshchuk et al. 2011) used a 1D approach to describe the distribution of an electrolyte in a micro-channel "plugged" by a micro-/nano-interface, in order to show that the hydrodynamic-dispersion mechanism becomes effective in sufficiently broad micro-channels (hundreds of micrometer in height). On the contrary, the surface-conductance mechanism explored in (Mani et al. 2009) is responsible for the appearance of deionization "shocks" in submicro-channels (commensurate with the screening length), and hence those two mechanisms operate under very different conditions. Therefore one of them can be disregarded when considering the other. Rubinstein and Zaltzman (Rubinstein and Zaltzman 2013) considered approximate analytical and numerical solutions of 2D convective-diffusion problem for an uncharged solute in a micro-channel evaluating various boundary conditions at the channel ends to explore the breakdown of the Taylor-Aris dispersion mechanism with increasing circulation velocity.

The EOC is important for microfluidics becuase it could be a way to reduce the CP near nanoporous media used in microfluidic systems, which results in the limiting and over-limiting current phenomena (Yaroshchuk et al. 2011; Dydek et al. 2011). To sum up, the studies of the solute-transport mechanisms in microfluidic systems involving EOC are important for understanding such phenomena as concentration polarization or concentration shock waves and the role of hydrodynamic dispersion in these phenomena. 
In this work we consider the steady-state distribution of an electrolyte in a micro-channel under EOC with imposed different concentrations at the ends of the channel. This is a continuation of the previous study (Part I, Licon Bernal et al. 2014), where the transport of a non-electrolyte species under similar conditions was considered. By imposing different concentrations of electrolyte at the channel ends, we simulate the situation of concentration polarization within the channel but disregard the complex phenomena of electro-hydrodynamic instability and/or water splitting known for occurring close to current-polarized interfaces between ion-exchange materials and electrolyte solutions. Having understood the behavior of such simplified system we plan to superimpose those phenomena and hydrodynamic dispersion in future studies.

Numerical simulations are compared with an approximate analytical description of the system. Furthermore, simple parametric relationships are derived to capture the principal features of the phenomena in particular the behavior of current vs voltage characteristics. One of the important conclusions of this study is that the Taylor-Aris dispersion theory is approximately applicable locally, within a major part of the channel, despite the considerable variation of EOC rate along the channel.

\section{Formulation of the problem and governing equations}

In the previous study (Licon Bernal et al. 2014a) (from here on this publication is referred to as Part I) we considered the convective-diffusion transport of a non-electrolyte in an open long channel with different time-independent solute concentrations at the ends in the presence of a buffer electrolyte, whose concentration was constant throughout the system. Due to this the velocity of electroosmotic flow at the channel walls did not vary along the channel being independent of the non-electrolyte distribution. Under such conditions, the hydrodynamics could be decoupled from the solute transfer.

In the present study we consider the transport of an electrolyte whose concentration changes along the channel. Therefore, the velocity of electroosmotic flow is not constant along the channel because it is dependent on the local electrolyte concentration. Hence, the hydrodynamics cannot be decoupled from the solute transfer now and the analysis becomes more complicated. In addition, when the electroosmotic flow velocity varies along the channel a transversal velocity component arises due to the flow continuity. The transversal velocity influences the concentration distribution within the channel cross-sections, thus, affecting the 
solute transfer. Nevertheless, as shown below, in this case, too, the Taylor-Aris dispersion theory turns out approximately applicable albeit locally.

For the numerical analysis, a binary electrolyte, electroneutrality and no diffusive contribution to the electric current is assumed. We will use the set of steady state convection-diffusion and Navier-Stokes equations coupled with the electric-current conservation law:

$$
\begin{aligned}
& \nabla \cdot(-D \nabla C)+\mathbf{u} \cdot \nabla C=0 \\
& \rho(\mathbf{u} \cdot \nabla) \mathbf{u}=\nabla \cdot(\mu \nabla \mathbf{u}-P) ; \quad \nabla \cdot \mathbf{u}=0 \\
& \nabla \cdot \boldsymbol{J}=0 ; \quad \boldsymbol{J}=\sigma \boldsymbol{E} ; \quad \boldsymbol{E}=-\nabla V
\end{aligned}
$$

where $D$ and $C$ are the electrolyte molecular diffusion coefficient and concentration, $\rho$ and $\mu$ are the solution density and dynamic viscosity, $P$ is the pressure, $\boldsymbol{u}=\left[\begin{array}{l}u_{x} \\ u_{y}\end{array}\right]$ is the velocity vector, $\nabla=\left[\begin{array}{ll}\frac{\partial}{\partial x} & \frac{\partial}{\partial y}\end{array}\right]$ is the gradient operator, $x$ and $y$ are the longitudinal and transverse coordinates, respectively, for a flat channel, $\boldsymbol{J}=\left[\begin{array}{c}j_{x} \\ j_{y}\end{array}\right]$ and $\sigma=\alpha C$ are the local electric current density and conductivity, $\alpha$ is the proportionality coefficient between the conductivity and concentration, and $\boldsymbol{E}$ and $V$ are the local electric field strength and electric potential.

To define the boundary conditions, first different constant concentrations at the channel ends and zero flux condition at the channel walls are set as:

$$
C(0, y)=C_{0} ; \quad C(L, y)=C_{L} ; \quad \boldsymbol{n} \cdot(-D \nabla C(x, \pm h)+\boldsymbol{u} C(x, \pm h))=0
$$

where $h$ and $L$ are the half-height and the length of the channel $(h \ll L)$.

The hydrodynamic boundary conditions at the edges of the channels are defined using the assumption of zero normal stress $\left(\frac{\partial u_{x}}{\partial x}=0\right)$, which is compatible with the picture of a channel connected to a big reservoir (Panton 2013), and the condition of exact compensation of crosssection-averaged Poiseuille and electroosmotic flows (Licon Bernal et al. 2014b):

$$
\begin{aligned}
& u_{x}(0, y)=\frac{u_{e o}(0)}{2}\left(\frac{3 y^{2}}{h^{2}}-1\right) \text { and } u_{y}(0, y)=0 \\
& u_{x}(L, y)=\frac{u_{e o}(L)}{2}\left(\frac{3 y^{2}}{h^{2}}-1\right) \text { and } u_{y}(L, y)=0
\end{aligned}
$$


The y-component condition follows from the conditions $\frac{\partial u_{x}}{\partial x}=0$ and the continuity equation $\left(\frac{\partial u_{x}}{\partial x}=-\frac{\partial u_{y}}{\partial y}\right)$. Such boundary conditions reflect the fact that there are no abrupt changes in the flow pattern at the two ends of the microchannel (Lee et al. 2005). Although the definition of the boundary condition for the hydrodynamics is non-trivial, below we will see that the concentration profile inside the channel does not appreciably change when using different boundary conditions at the channel ends. This is due to the constant concentrations set at these boundaries.

The slip condition holds for the velocity at the channel walls, defined as the velocity of electroosmotic flow found from the Helmholtz-Smoluchowski equation

$u_{e o}(x)=u_{x}(x, \pm h)=-\frac{\varepsilon \varepsilon_{0} \zeta}{\mu} E_{x}(x, \pm h)$

where $\varepsilon$ is the relative dielectric constant, $\varepsilon_{0}$ is the dielectric permittivity of vacuum, and $\zeta$ is the zeta-potential at the walls. The electroosmotic flow superimposed with a pressure-driven counter-flow produces the characteristic flow field of electroosmotic circulation. Similarly to the case in Part I, we assume here that the electroosmotic flow and the pressure-driven flow exactly compensate each other on average giving rise to zero net volume flow through the channel (see Eq.(5)).

Finally, the current density is set constant at the channel edges, while the channel walls are considered insulating

$\boldsymbol{n} \cdot \boldsymbol{J}(0, y)=-\boldsymbol{n} \cdot \boldsymbol{J}(L, y)=J_{0} ; \quad \boldsymbol{n} \cdot \boldsymbol{J}(x, \pm h)=0$

We will assume here that electro-neutrality condition holds everywhere within the electrolyte solution, except of infinitely thin layers at the channel walls. Eqs.(3) and (7) show that the total electric current through each channel cross-section must be constant. Though we consider channel walls with constant zeta-potential, the electroosmotic flow velocity varies along the channel because of the varying electric-field strength due to the changing solution conductivity (related to the changing electrolyte concentration).

The 2D model has been solved by Finite Element method (FEM) with COMSOL Multiphysics 4.2a to describe the concentration distribution of a salt $(\mathrm{KCl})$ dissolved in water inside a flat microchannel. In the numerical simulations, various values of electric current $(I)$ passing through the 
channel have been evaluated. The local conductivity, $\sigma$, was calculated in the ideal-solution approximation with the respective diffusion coefficients for the ions $\left(\mathrm{K}^{+}\right.$and $\left.\mathrm{Cl}^{-}\right)$.

The first step was to declare the domain as a rectangle of the length $L$ and the height two times $h$. A fixed value of $h=100 \mu \mathrm{m}$ was used for all the calculations, while the channel lengths $(L)$ was varied. In most cases the concentrations at the channel edges were set to $C_{0}=1 \mathrm{~mol} / \mathrm{m}^{3}$ and $C_{L}=10 \mathrm{~mol} / \mathrm{m}^{3}$, some other combinations were considered, too, with the purpose of evaluating their effect. A free triangular mesh was created with a refinement on the walls of the micro-channel with a minimum element size of 1 micrometer. The equations were solved with the parallel direct linear solver (PARDISO) with a relative tolerance of 0.001 .

\section{TAD approach to the description of solute transport within a long straight channel with a transversal velocity component}

The classical Taylor-Aris analysis assumed the fluid-velocity profile to be the same everywhere in the channel. In our problem, the rate of EOC can change considerably along the channel due to the changes of electroosmotic slip velocity caused by the variation of solution conductivity. Despite this variation, in (Yaroshchuk et al. 2011) it was postulated that the TAD approach was locally applicable for the description of hydrodynamic dispersion under conditions of concentration polarization in a long narrow channel. The purpose of this section is to investigate the applicability of the approach of effective diffusion coefficient under conditions of spatially-variable flow-velocity profile by means of a rigorous 2D analysis.

For a steady-state convection-diffusion solute transfer in a flat channel Eq.(1) can be written as

$$
-u_{x} \frac{\partial C}{\partial x}-u_{y} \frac{\partial C}{\partial y}+D \frac{\partial^{2} C}{\partial x^{2}}+D \frac{\partial^{2} C}{\partial y^{2}}=0
$$

In the previous study (Part I) we considered the case of convective-diffusion transport of a nonelectrolyte. The concentration of buffer electrolyte was assumed to be constant throughout the system, so the electroosmotic velocity was constant along the channel and the transversal velocity component was absent. In the present study we consider the transport of an electrolyte, whose concentration is varying along the channel. Therefore, the longitudinal velocity is varying, too, and, according to the flow-continuity condition the transversal velocity component should be non-zero

$$
\frac{\partial u_{x}}{\partial x}=-\frac{\partial u_{y}}{\partial y} \neq 0
$$


This results in the appearance of the second term in Eq.(8). Thus, the convective flow contributes to the solute distribution within the channel cross-sections and, therefore, can influence the overall solute transport through the channel. This is the principal difference of the present system from the conventional case of Taylor-Aris Dispersion (Yaroshchuk et al. 2011; Rubinstein and Zaltzman 2013), where the transversal convection is absent.

Proceeding in the same way, as in the previous study, for a straight long channel $(h<L)$ we will represent the local concentration and velocity components as sums of cross-section averaged values and deviations from the average

$C(x, y)=\bar{C}(x)+\delta C(x, y) ; \quad u_{x}(x, y)=\bar{u}_{x}(x)+\delta u_{x}(x, y) ; \quad u_{y}(x, y)=\bar{u}_{y}(x)+\delta u_{y}(x, y)$

with $\bar{C}(x)=\frac{1}{2 h} \int_{-h}^{h} C(x, y) d y ; \bar{u}_{x}(x)=\frac{1}{2 h} \int_{-h}^{h} u_{x}(x, y) d y ; \bar{u}_{y}(x)=\frac{1}{2 h} \int_{-h}^{h} u_{y}(x, y) d y$. Then taking the cross-section average of Eq.(8) one obtains

$D \frac{d^{2} \bar{C}}{d x^{2}}-\frac{d\left(\overline{\delta u_{x} \delta C}\right)}{d x}-\bar{u}_{x} \frac{d \bar{C}}{d x}+\overline{\delta C \frac{\partial\left(\delta u_{x}\right)}{\partial x}}-\bar{u}_{y} \frac{\overline{\partial(\delta C)}}{\partial y}-\overline{\delta u_{y} \frac{\partial(\delta C)}{\partial y}}=0$

Subtracting Eq.(11) from the initial Eq.(8) one obtains:

$D \frac{\partial^{2} \delta C}{\partial x^{2}}+D \frac{\partial^{2} \delta C}{\partial y^{2}}+\frac{d\left(\overline{\delta u_{x} \delta C}\right)}{d x}-\overline{u_{x}} \frac{\partial \delta C}{\partial x}-\delta u_{x} \frac{d \bar{C}}{d x}-\delta u_{x} \frac{\partial \delta C}{\partial x}-\overline{\delta C \frac{\partial \delta u_{x}}{\partial x}}-\overline{u_{y}} \frac{\partial \delta C}{\partial y}-\delta u_{y} \frac{\partial \delta C}{\partial y}+\overline{u_{y}} \frac{\overline{\partial(\delta C)}}{\partial y}+\overline{\delta u_{y} \frac{\partial(\delta C)}{\partial y}}=0$

Eqs.(11) and (12) represent a set of two equations for the averaged concentration $\bar{C}(x)$ and the deviation $\delta C(x, y)$.

Considering large aspect ratios, $\lambda=L / 2 h>>1$, one can represent the deviation of concentration from the average as a series in the powers of the small parameter $\xi=h / L$, which is half the reciprocal aspect ratio

$\delta C=\delta C^{(0)}+\xi \delta C^{(1)}+\xi^{2} \delta C^{(2)}+\ldots$

Substituting this expansion into Eq.(12) and retaining only the zero-order terms in $\xi$, it is easy to show that $\delta C^{(0)}=0$ (see Appendix A). For the first-order term one obtains $\delta C^{(1)}=-P e \cdot \omega \cdot \frac{d \bar{C}}{d \tilde{x}}$, where $P e=h u_{c} / D$ is the Péclet number, $u_{C}$ is a characteristic velocity, which can be chosen, e.g. as $u_{C}=u_{e o}(0), \omega=\omega(\tilde{x}, \tilde{y})$ is a dimensionless function defined by the velocity distribution, $\tilde{x}=x / L$ and $\tilde{y}=y / h$ are the dimensionless coordinates (see Appendix A). Thus, within this approximation the concentration deviation from the average is proportional to the local average-concentration gradient along the channel, which means that the Taylor-Aris dispersion concept is applicable locally in this case. It should be noted, however, that this solution is valid 
only for an inner part of the channel away from the edge boundary layers because at the channel ends the concentration is assumed to be constant, which corresponds to $\delta C=0$.

With the concentration deviation given by $\delta C \approx \xi \delta C^{(1)}$ the equation for the average concentration, Eq.(11), takes this form (see Appendix A)

$$
\frac{d}{d \tilde{x}}\left[\left(1+P e^{2} \overline{\delta \tilde{u}_{x} \omega}\right) \frac{d \bar{C}}{d \tilde{x}}\right]=0
$$

Eq.(14) is a conventional stationary diffusion equation with an effective diffusion (dispersion) coefficient, $D_{T}=D\left(1+P e^{2} \overline{\delta \tilde{u}_{x} \omega}\right)$. This is in agreement with the Taylor-Aris dispersion model. The dispersion coefficient $D_{T}$ takes into account the effect of convection on the solute transfer through the channel. For large Péclet numbers $(P e>>1)$ the solute transfer is controlled mainly by the hydrodynamics. In the case of non-electrolyte transfer the velocity field within the channel does not depend on the solute concentration so the dispersion coefficient $D_{T}$ is constant. This leads to a linear longitudinal concentration profile (Part I). In the case of electrolyte solution with varying concentration the electroosmosis velocity depends on the local concentration and, thus, the dispersion coefficient $D_{T}$ is also a function of concentration. Consequently, the solution of Eq.(14) should give a non-linear longitudinal profile of average concentration. Similarly to the equation for the concentration deviation, Eq.(A.6), Eq.(14) describes the average-concentration distribution only within an inner part of the channel and is not applicable to the boundary layers close to the channel ends where the full initial set of Eqs.(1)-(3) should be solved.

We consider here a superposition of pressure-driven and electroosmotic flows that compensate each other on average so that the net volume flow through the channel is zero. For long narrow channels the longitudinal velocity can be approximately represented as a sum of Poiseuille and plug-like flows (Appendix B)

$$
u_{x}=\frac{u_{e o}(x)}{2}\left(\frac{3 y^{2}}{h^{2}}-1\right), \quad \bar{u}_{x}=0
$$

where the velocity of electroosmotic flow at the walls, $u_{e o}(x)$, is given by Eq.(6).

For the velocity profile given by Eq.(15), the integration of Eq.(A.7) gives $\omega=\widetilde{u}_{e o}(x) \cdot\left(-\frac{7}{120}+\frac{\tilde{y}^{2}}{4}-\frac{\tilde{y}^{4}}{8}\right)$, where $\tilde{u}_{e o}=u_{e o}(x) / u_{C}=u_{e o}(x) / u_{e o}(0)$ is the dimensionless velocity normalized by the characteristic (electroosmotic) velocity at the channel entrance $\left(u_{c}=u_{e o}(0)\right)$. Then for the dispersion coefficient one has $D_{T}=D \cdot\left(1+\frac{2}{105} P e^{2} \tilde{u}_{e o}^{2}\right)$ or 
$D_{T}=D \cdot\left(1+\frac{2}{105} \frac{h^{2} u_{e o}^{2}}{D^{2}}\right)$ with $P e \tilde{u}_{e o}=\frac{h u_{e o}}{D}$ serving as a local Péclet number. This expression for the dispersion coefficient formally coincides with that given by the TAD model, but now it depends on the electroosmotic velocity, which varies along the channel. Thus the Taylor-Aris approach is approximately applicable in the present case (though only locally) because the transversal convection contributes only to the higher order terms in the series given by Eq.(13) as shown below. With this dispersion coefficient Eq.(14) takes this form

$$
\frac{d}{d x}\left[\left(1+\frac{2}{105} \frac{h^{2} u_{e o}^{2}}{D^{2}}\right) \frac{d \bar{C}}{d x}\right]=0
$$

The first integration of this equation gives

$$
\left(1+\frac{2}{105} \frac{h^{2} u_{e o}^{2}}{D^{2}}\right) \frac{d \bar{C}}{d x}=-\frac{Q}{D}
$$

where $Q$ is the solute flux through the channel, which is constant along the channel under steady-state conditions.

For the second integration we have to know the dependence of electroosmotic velocity on the concentration. By neglecting the variation of $x$-component of electric field within the channel cross-section (which is negligibly small according to our estimates and calculations, see below Sub-section 4.2) we can write approximately $E_{x}(x, \pm h)=\frac{I}{\alpha \bar{C}(x)}$, where $I$ is the average current density and $\bar{C}(x)$ is the average concentration. Then, according to Eq.(6), the electroosmotic flow velocity is inversely proportional to the cross-section-averaged electrolyte concentration

$$
u_{e o}(x)=\frac{\chi}{\bar{C}(x)}
$$

where $\chi=-\frac{\varepsilon_{0} \zeta I}{\mu \alpha}$ is the proportionality coefficient. The direction of electroosmotic flow depends on the direction of current and sign of zeta-potential at the walls. By using Eq.(18)), from $\mathrm{Eq}(17)$ we obtain this longitudinal concentration profile

$$
D(\bar{C}(x)-\bar{C}(0))-\frac{2 P e^{2}}{105} D \bar{C}^{2}(0)\left(\frac{1}{\bar{C}(x)}-\frac{1}{\bar{C}(0)}\right)=-Q x
$$

and therefore

$$
Q=-\frac{D}{L}(\bar{C}(L)-\bar{C}(0))+\frac{2 P e^{2}}{105} \frac{D \bar{C}^{2}(0)}{L}\left(\frac{1}{\bar{C}(L)}-\frac{1}{\bar{C}(0)}\right)
$$


This result shows that for small to moderate Pe the average-concentration profile in the channel is close to linear, whereas for large Péclet numbers the linear dependence occurs for the reciprocal average concentration. Note, however that this solution was obtained for the inner part of the channel, away from the boundary layers at its ends. Below we will see that the $\bar{C}(0)$ and $\bar{C}(L)$ actually mean average concentrations extrapolated from the inner part of the channel to the external channel edges and do not coincide with the external concentrations $C_{0}$ and $C_{L}$ (see Section 4).

Using the obtained function $\omega(\tilde{x}, \tilde{y})$ one can find the concentration deviation from the average for the inner part of the channel

$$
\delta C(\tilde{x}, \tilde{y})=\xi \cdot P e \cdot \tilde{u}_{e o}(\tilde{x}) \cdot \frac{d \bar{C}}{d \tilde{x}} \cdot\left(\frac{7}{120}-\frac{\tilde{y}^{2}}{4}+\frac{\tilde{y}^{4}}{8}\right)
$$

This approximate solution shows that the Taylor-Aris dispersion model is applicable locally within an inner part of the channel. Accounting for the higher-order terms in the reciprocal aspect ratio results in deviations from the local Taylor-Aris approach. It should be taken into account in the case of channels with not very large aspect ratios.

As discussed above due to the dependence of longitudinal velocity on $x$, there is a non-zero transversal velocity. One can estimate this transversal velocity by integrating Eq.(9) with the account of Eq. (15) and condition $u_{y}(x, 0)=0$

$$
u_{y}=\frac{d u_{e o}}{d x} \cdot \frac{y}{2} \cdot\left(1-\left(\frac{y}{h}\right)^{2}\right)
$$

Then the second-order term for the concentration deviation from the average, $\delta C^{(2)}$, is given by Eq.(A.11) in Appendix A. With the account for this term Eq.(11) for the average concentration transforms to

$\frac{d}{d \tilde{x}}\left[\frac{d \bar{C}}{d \tilde{x}}+\frac{2 P e^{2} \tilde{u}_{e o}^{2}}{105} \frac{d \bar{C}}{d \tilde{x}}-\xi \frac{2 P e^{3} \tilde{u}_{e o}^{2}}{105 \cdot 55} \frac{d \tilde{u}_{e o}}{d \tilde{x}} \frac{d \bar{C}}{d \tilde{x}}-\xi \frac{4 P e^{3} \tilde{u}_{e o}^{3}}{105 \cdot 165} \frac{d^{2} \bar{C}}{d \tilde{x}^{2}}\right]=0$

The first two terms in the square brackets in Eq.(23) are the same as in the local Taylor-Aris approximation (cf. Eq.(16)), whereas the last two terms are corrections to this model. They increase with increasing $\xi$ (decreasing aspect ratio) and Péclet number. Integrating Eq.(23) one obtains the solute flux through the channel

$$
\tilde{Q}=\frac{Q L}{D}=-\left(1+\frac{2 P e^{2} \tilde{u}_{e o}^{2}}{105}(1+k)\right) \frac{d \bar{C}}{d \tilde{x}}
$$

where the function $k$ is given by 


$$
k=-\xi \frac{P e}{55} \frac{d \tilde{u}_{e o}}{d \tilde{x}}-\xi \frac{2 P e \tilde{u}_{e o}}{165} \frac{d^{2} \bar{C}}{d \tilde{x}^{2}}\left(\frac{d \bar{C}}{d \tilde{x}}\right)^{-1}
$$

Transforming to the dimensional form and substituting $u_{e o} \approx \chi / \bar{C}$, one obtains

$$
k=\frac{h^{2} \chi}{55 D \bar{C}^{2}} \frac{d \bar{C}}{d x}-\frac{2 h^{2} \chi}{165 D \bar{C}} \frac{d^{2} \bar{C}}{d x^{2}}\left(\frac{d \bar{C}}{d x}\right)^{-1}
$$

In the derivation of function $k$, given by Eq.(26), we used the approximate velocity field, given by Eq.(15) and (22) instead of solving the Navier-Stokes equations. However, it can be shown that this gives rise only to minor corrections for our system (see Appendix B).

The first and second derivatives of average concentration are always positive, (see below), so the terms in Eq.(26) have opposite signs and partially compensate each other. However, since both these terms include different combinations of average-concentration derivatives, their relative contributions change along the channel. As a result the function $k$ depends on the longitudinal coordinate and can change sign.

As discussed above for small to moderate Péclet numbers the average concentration profile is linear

$$
\bar{C}(x)=\bar{C}(0)+(\bar{C}(L)-\bar{C}(0)) \frac{x}{L}
$$

and the function $k$ is given by

$$
k=\frac{h^{2} \chi L}{55 D} \frac{\bar{C}(L)-\bar{C}(0)}{(\bar{C}(0) L+(\bar{C}(L)-\bar{C}(0)) x)^{2}}
$$

For large Péclet numbers the linear dependence occurs for the reciprocal average concentration

$$
\frac{1}{\bar{C}(x)}=\frac{1}{\bar{C}(0)}+\left(\frac{1}{\bar{C}(L)}-\frac{1}{\bar{C}(0)}\right) \frac{x}{L}
$$

For such concentration profile one obtains

$$
k=-\frac{h^{2} \chi}{165 D} \frac{\bar{C}(L)-\bar{C}(0)}{\bar{C}(0) \bar{C}(L) L}
$$

Thus, the correction to the Taylor-Aris model has opposite signs for small and large Péclet numbers. For intermediate Péclet numbers the correction can change sign along the channel. Its behavior will be considered in more detail below, in the section 4.4. 


\section{Results and Discussion}

\subsection{Electrolyte concentration distribution within the micro-channel}

An example of numerical solutions for the electrolyte distribution under conditions of EOC in a micro-channel and the corresponding velocity field is shown in Figure 1. The local magnitude of the velocity at the walls is inversely proportional to the electrolyte concentration. Therefore it changes from one cross-section to another while preserving the same characteristic shape. This is a superposition of electroosmotic and pressure-driven counter-flows with zero cross-sectionaveraged velocity. Such velocity field gives rise to a transversal non-uniformity in the concentration distribution which will be studied below.

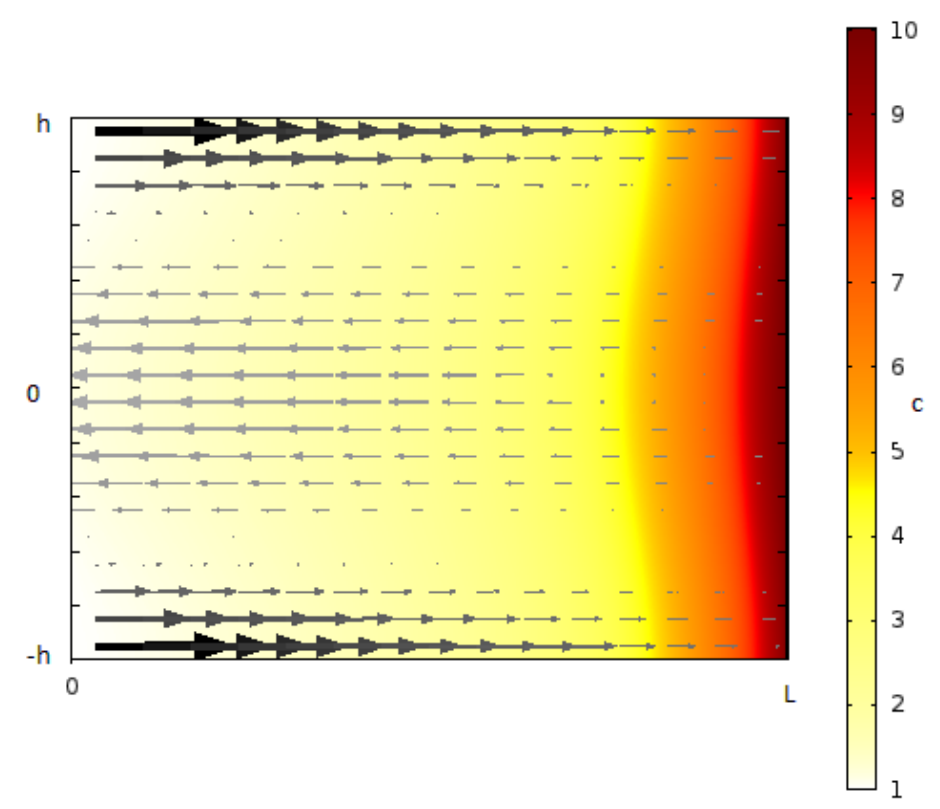

Figure 1. Velocity field (arrows) and electrolyte concentration, $\mathrm{mol} / \mathrm{m}^{3}$ (color), in a channel of 4 $\mathrm{mm}$ length and half-height $h=100 \mu \mathrm{m}$ for the current density $I=250 \mathrm{~A} / \mathrm{m}^{2}\left(u_{e o}>0\right)$. (color figure online)

Figure 2 shows how the averaged-concentration profile depends on the current density. A nonlinear concave-down shape occurs for all the curves and this non-linearity becomes more pronounced with increasing current density. The non-linear shape is a consequence of nonuniformity of electroosmotic velocity. The velocity is higher in the left part of the channel, and, therefore, the dispersion coefficient $D_{T}=D \cdot\left(1+\frac{2}{105} \frac{h^{2} u_{e o}^{2}}{D^{2}}\right)$ is larger here, too. To maintain the same solute flux, this is compensated by a smaller concentration gradient. On the right side the velocity and the dispersion coefficient are smaller, and the concentration gradient is larger. 
On the "dilute" side a small concentration "jump" in the ordinate axis can be seen (Figure 2). On the "concentrated" side a "jump" is much less pronounced. With increasing current density the "jumps" of the cross-section averaged concentration at the borders are increasing. As shown in Figure 1, depending on the height of a given longitudinal cross section, the flow has one direction or another. For this case of study, the liquid moves towards the diluted concentration side (to the left) at the middle height channel region and towards the concentrated side (to the right) at the channel walls. It strongly affects the local concentration, i.e. the outflowing solution hits the boundary and in order to satisfy the boundary condition, the local concentration profile changes abruptly and such effect is captured when making a height cross sectional averaging (profiles shown in Figure 2). On the other hand, at the same side over the channel wall the concentration at the diluted side is equal to the boundary condition even some distance inside due to the incoming solution. Such zone will be referred in this study as boundary layer.

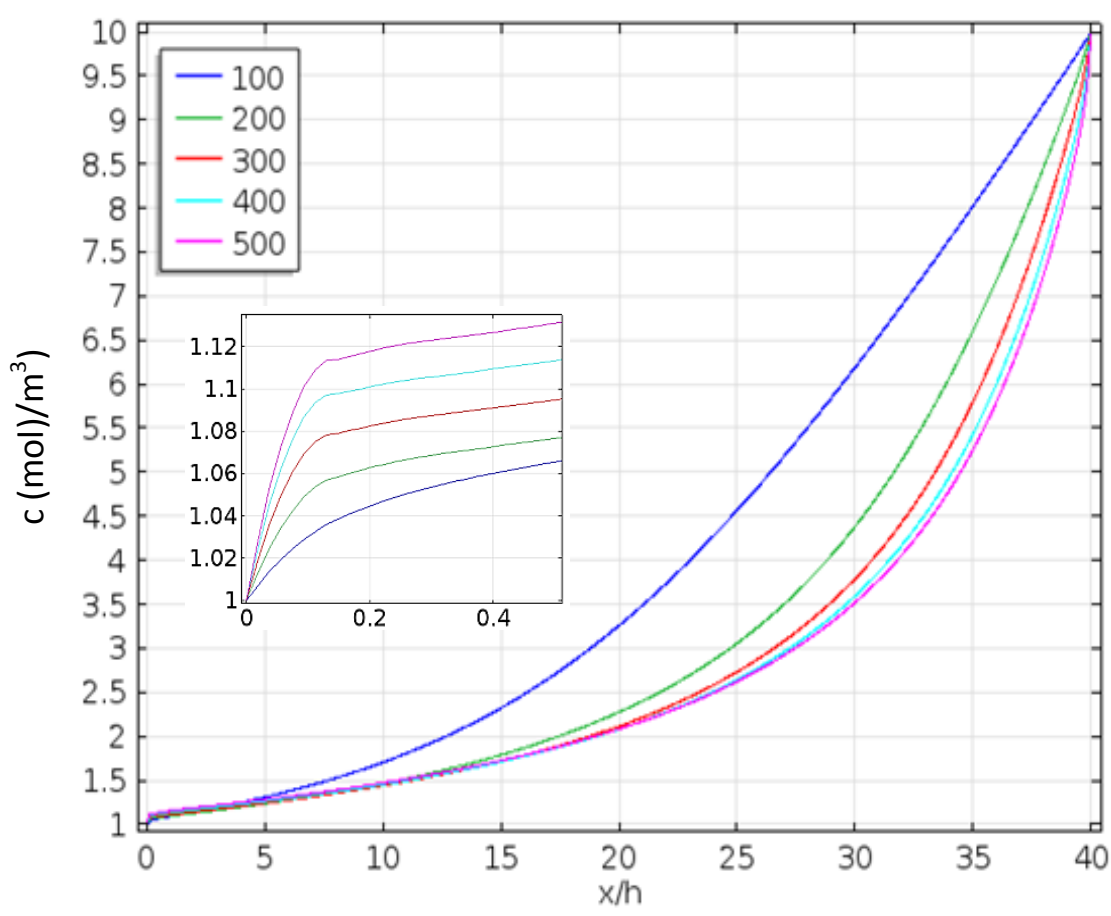

Figure 2. Averaged concentration profiles in a channel of $4 \mathrm{~mm}$ length and half-height $h=100$ $\mu \mathrm{m}$ for various values of current density: $100,200,300,400,500 \mathrm{~A} / \mathrm{m}^{2}\left(u_{e o}>0\right)$. Inset represents a zoomed area near to the channel edge with low concentration.

The length of the boundary layers increases with current density at the channel wall according to Figure 3. Qualitatively similar boundary layers were observed in the study of mass transfer of a non-electrolyte considered in Part I. The electric-field strength is proportional to current 
density and inversely proportional to conductivity, so, according to Eq.(6), the electroosmotic velocity is inversely proportional to the concentration. In Figure 3 the reciprocal-concentration profiles are shown for the same current densities, as in Figure 2. For the higher current densities, these profiles become close to linear. Such a transformation of concentration profile with increasing current density is in agreement with the approximate analytical solution obtained above (cf. Eq.(19)), and shows that the Taylor-Aris approach is approximately applicable to this system. However, there are boundary zones at the channel edges where this behavior is not observed, as it was also the case with the non-electrolyte transfer (Part I).

In order to compare these two systems, longitudinal local-concentration profiles along the channel for three different transversal positions $(y=0, h / \sqrt{3}$ and $h)$ are plotted in Figure 4 . Different slopes for these three locations occur because the magnitude of concentration variation within the channel cross-section increases with decreasing average concentration and increasing EOC rate. In contrast, in the case of non-electrolyte the profiles were running parallel due to the constant buffer electrolyte concentration, conductivity and EOC rate in the channel.

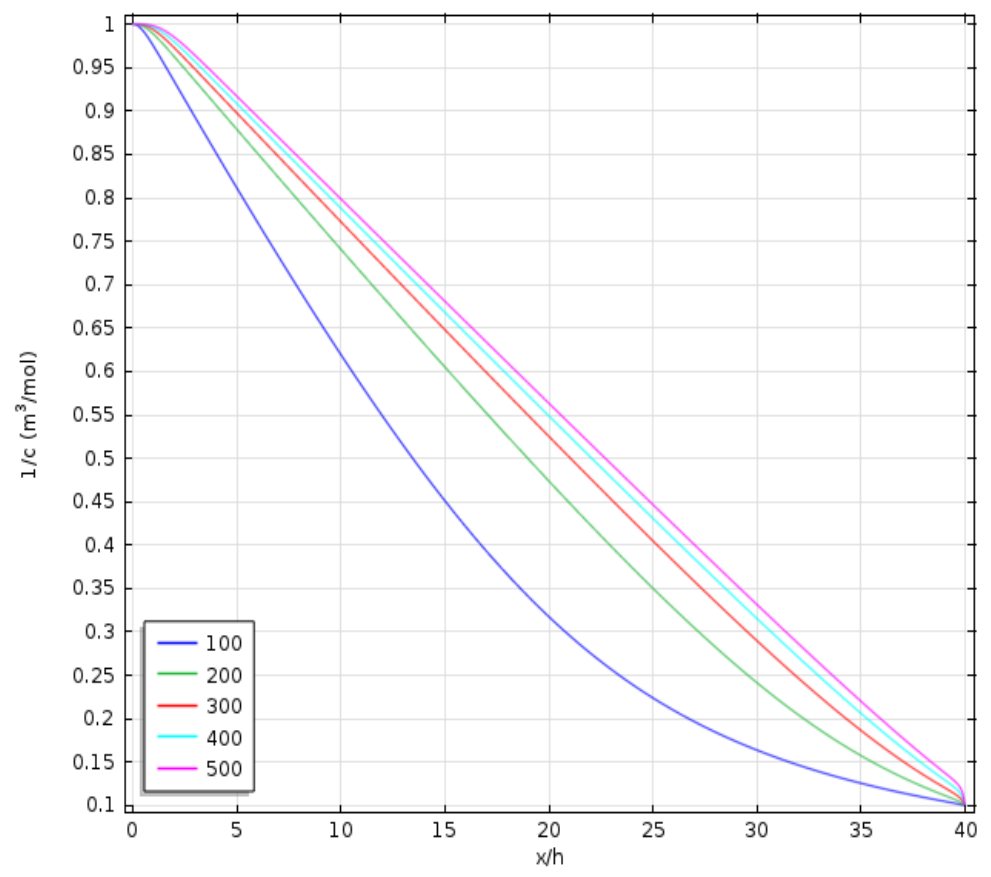

Figure 3. Profiles of local reciprocal concentration over the channel wall for various values of current density (in $\mathrm{A} / \mathrm{m}^{2}$ ) for the channel of $4 \mathrm{~mm}$ length and half-height of $h=100 \mu \mathrm{m}\left(u_{e o}>0\right)$. Since the intensity of convection is dependent on the electric field, the boundary zone (where the concentration stays nearly constant) near the channel edge with more dilute solution ( $x=$ 0 ) is more pronounced than on the opposite side. Besides, as in the case of non-electrolytes, 
the length of this zone is strongly dependent on the transversal coordinate, $y$. The boundary zone is very short in those parts of the cross-section where the solution leaves the channel (at the dilute side for $u_{e o}>0$ this is near the middle plane, $y=0$, and at the opposite side - near the walls, $y \approx \pm h$ ), and it is much longer at the locations, where the solution enters the channel $(y \approx$ $\pm h$ at the dilute side and $y \approx 0$ at the opposite side).

Figure 5 is a contour plot of the reciprocal concentration. It shows the evolution of profile shape from the constant concentrations at the channel edges to the Taylor-Aris type profiles in the inner part. This profile is defined by the $y$-dependence of concentration deviation given by Eq. (21).
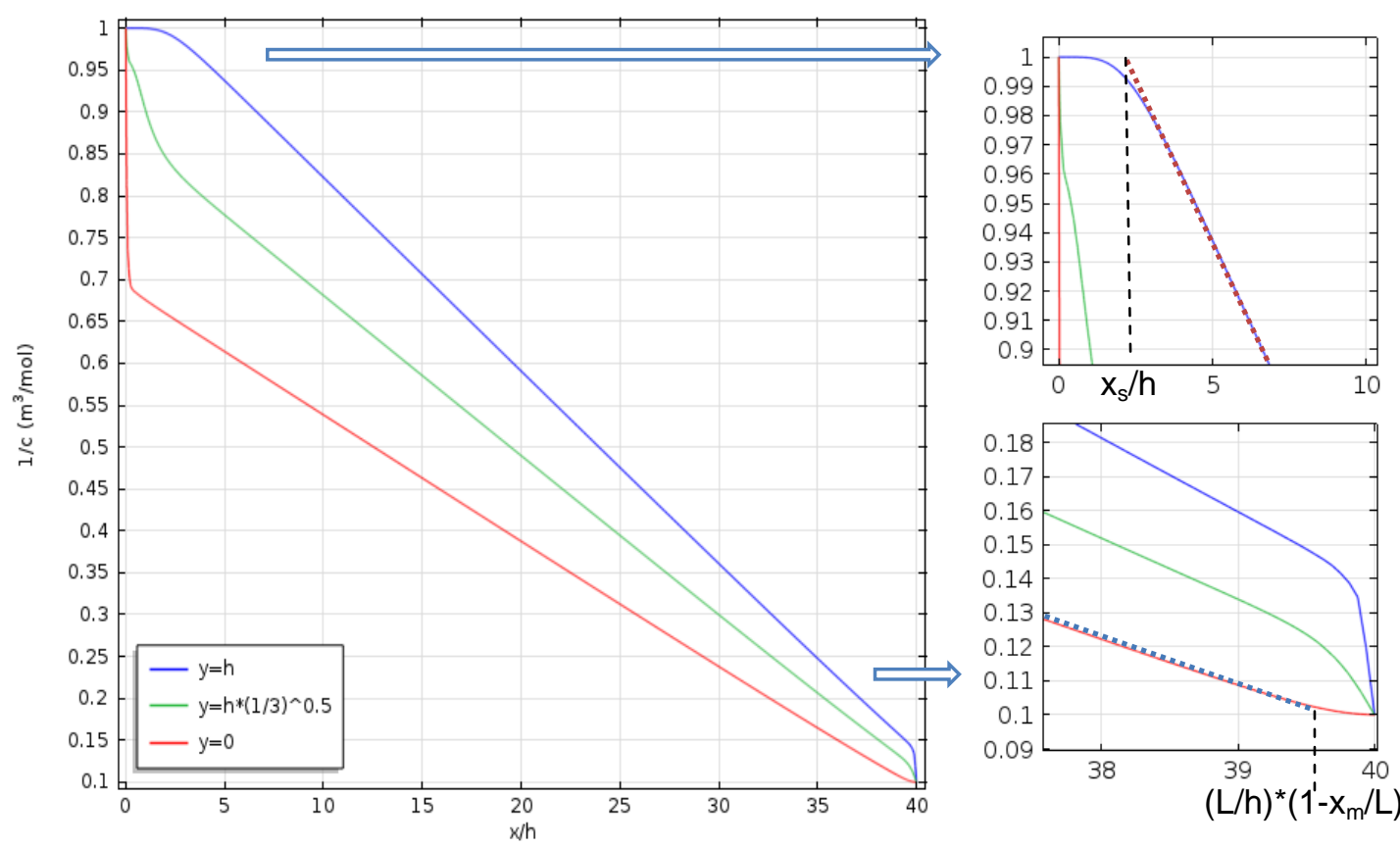

Figure 4. Profiles of reciprocal local concentration along the channel for various longitudinal cross-sections at the current density of $800 \mathrm{~A} / \mathrm{m}^{2}$ for the channel of $4 \mathrm{~mm}$ length and halfheight of $h=100 \mu \mathrm{m}\left(u_{e o}>0\right)$.

Figures 4 and 5 show that on the left channel side (close to $x=0)$ in the vicinity of the walls $(y \approx$ $\pm h$ ), there are extended zones where the concentration almost does not change remaining practically the same as at the edge. These zones arise due to the intrusions of external solution along the walls. A similar (albeit shorter) intrusion zone occurs also on the right side of the channel $(x=L)$, but in the vicinity of the middle plane $(y \approx 0)$. Such intrusions of the external solutions result in distortions of transversal concentration profiles compared to the inner part 
of the channel. Around the internal boundaries of these regions $\left(x=x_{s}\right.$ and $\left.x=L-x_{m}\right)$ the concentration profiles transform gradually to those corresponding to the TAD theory, Eq. (21). Similar effects within entrance zones of the channel were also observed in the case of nonelectrolyte transfer considered in the Part I of this study.

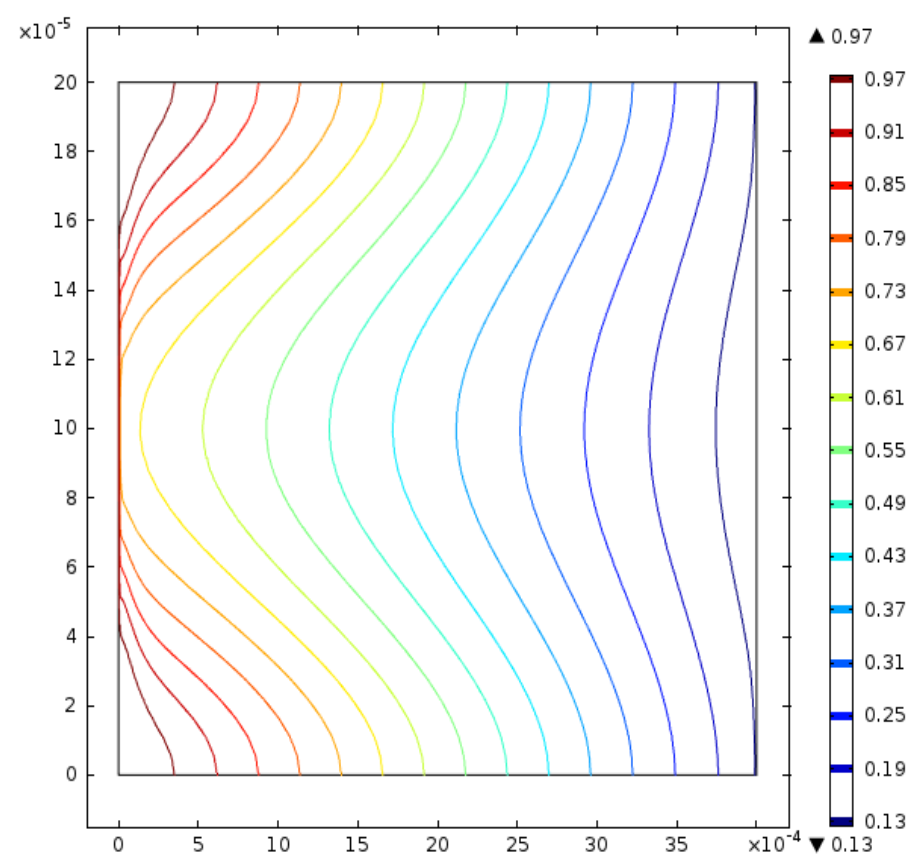

Figure 5. Contour graph showing the distribution of the reciprocal concentration within the channel at the current density of $800 \mathrm{~A} / \mathrm{m}^{2}$ for the channel of $4 \mathrm{~mm}$ length and half-height $h=$ $100 \mu \mathrm{m}$ (the scale for the distance in $x$ and $y$ directions is in meters, $u_{e o}>0$ ).

\subsection{Electric field distribution}

In the analytical derivations above, we assumed that the electric field is $1 \mathrm{D}$ within the inner part of the channel and the longitudinal electric-field component is independent of the transversal coordinate. The purpose of this subsection is to verify this assumption numerically.

Figure 6 shows the ratio of transversal and longitudinal electric-field components. Inside the channel the transversal field component is negligible compared to the longitudinal. The transversal component increases only very close to the channel edges but still remains very small. The ratio of the electric field components depends on the particular concentration distribution within the channel. In Appendix $C$ it is shown that, in the limit of high Peclet numbers, the concentration distribution represented by Eqs. (10a), (21) and (29) gives exactly zero transversal component and longitudinal component independent of the coordinate $y$. 
Numerical calculations confirm that $E_{y}$ is negligibly small and $E_{x}$ is almost independent of the coordinate $y$ also for not very high Peclet numbers.

An approximately uniform (within the channel cross-section) 1D electric field allows assuming the inverse proportionality of the electroosmotic velocity to the average concentration, $u_{e o} \approx \chi / \bar{C}$ (see Eq.(18)). The values of $\chi \approx u_{e o} \bar{C}$ calculated numerically are practically constant. For example, for the case of a microchannel under $100 \mathrm{~A} / \mathrm{m}^{2}$ and $C_{L} / C_{0}=100$, the variation of this value along the channel (away from the entrance boundary layers) is less than $0.1 \%$. Moreover calculations show a linear dependence of $\chi \approx u_{e o} \bar{C}$ on the current density.

a)
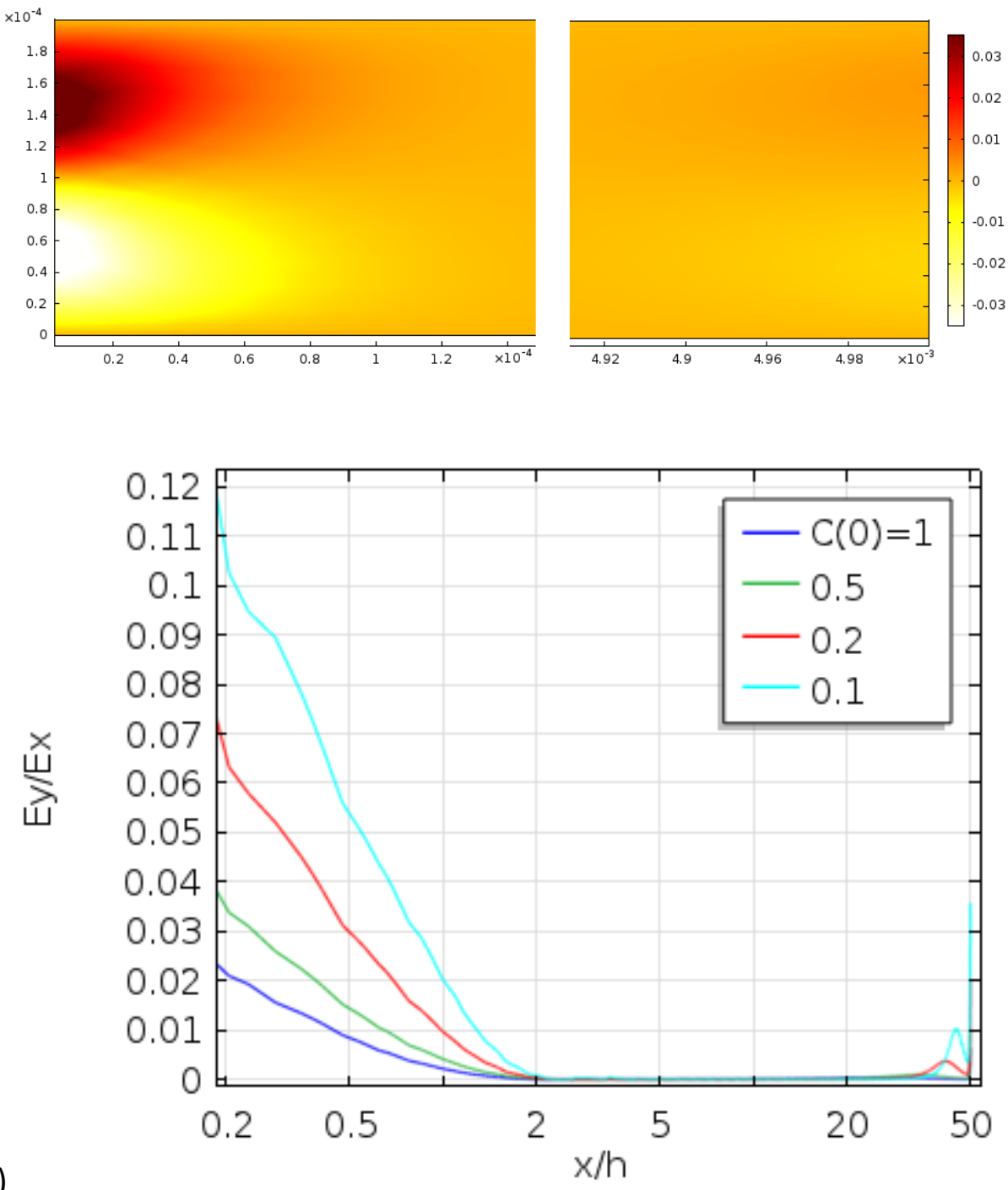

Figure 6. Ratio of the transversal and longitudinal electric field components, $E_{y} / E_{x}$, at $100 \mathrm{~A} / \mathrm{m}^{2}$ of current density inside the micro-channel of $5 \mathrm{~mm}$ length and $100 \mu \mathrm{m}$ of half-height for a concentration ratio $C_{L} / C_{0}=100$ (a) and variation of this ratio along the channel at $y=h^{*}(1 / 3)^{1 / 2}$ for the concentration ratios 10, 20, 50 and 100 (b) ( $\left.u_{e o}>0\right)$. (color figure online) 


\subsection{Hydrodynamics}

The profile of longitudinal velocity in the middle cross-section $(x=L / 2)$ of the channel is presented in Figure 7a. The absolute value of the velocity is shown in order to see better graphically the points where the velocity changes the direction. This profile is the result of superposition of a parabolic (pressure-driven) and an electroosmotic (plug) flows. The velocity is zero at $\tilde{y}= \pm 1 / \sqrt{3}$, in agreement with Eq.(15). According to Eq.(22), the same points correspond to the largest absolute values of transversal component of velocity arising due to the flow continuity, Figure 7b. From Fig.7 one can also see that the transversal velocity component is about two orders of magnitude smaller than the longitudinal one.

Due to the changes in the conductivity along the channel, the magnitude of velocity variation across the channel cross-section changes but the shape of profile remains the same (except the edge boundary layers). In Figure 8 the length of the arrows represents the relative magnitude of velocity for various cross sections along the channel. The lines in this figure represent the locations within the cross-sections where the direction of liquid flow changes to the opposite and where the transversal component is the largest.

The rate of convection is largest near the left edge $(x=0)$ because the electrolyte concentration is the smallest here. Near the edge the arrows are not plane-parallel but have a tendency to deviate towards the middle of the channel because the concentration distribution within the boundary layers is strongly different from the inner part of the channel.
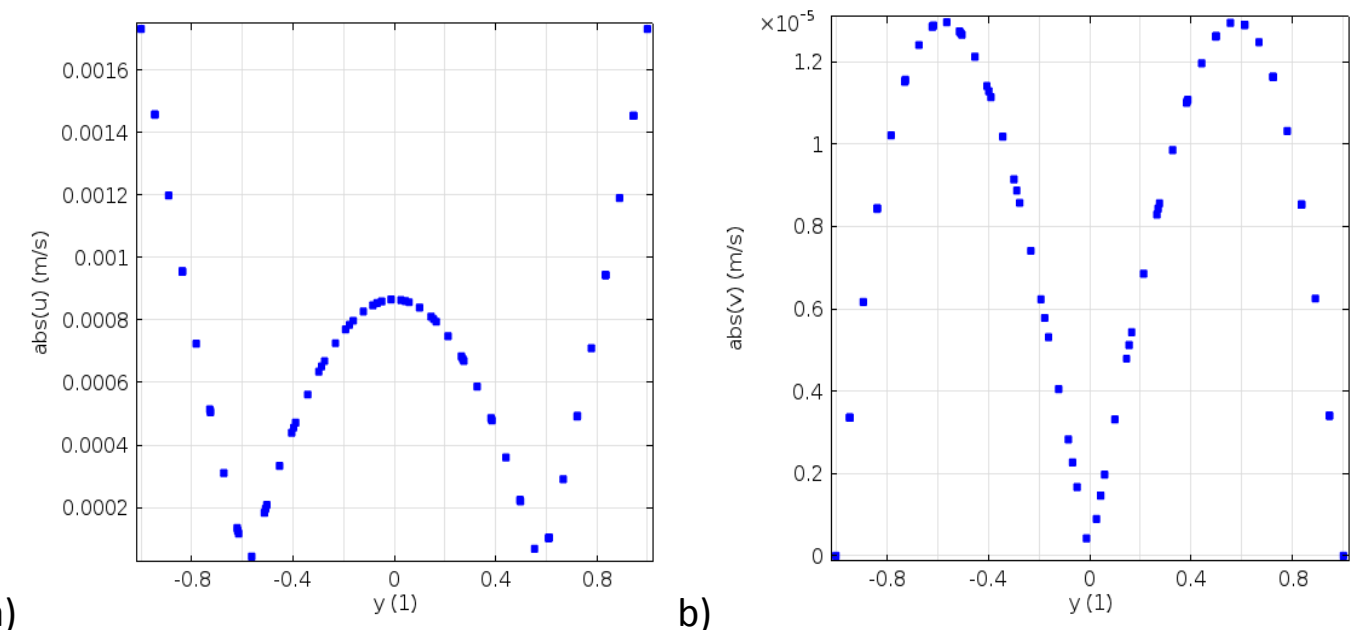

Figure 7. Velocity profiles at $800 \mathrm{~A} / \mathrm{m}^{2}$ at the middle cross-section of the channel $(x=L / 2)$ for a) x-component and b) y-component; channel length $L=4 \mathrm{~mm}$, half-height $h=100 \mu \mathrm{m}$, concentration ratio $C_{L} / C_{0}=10\left(u_{e o}>0\right)$. 


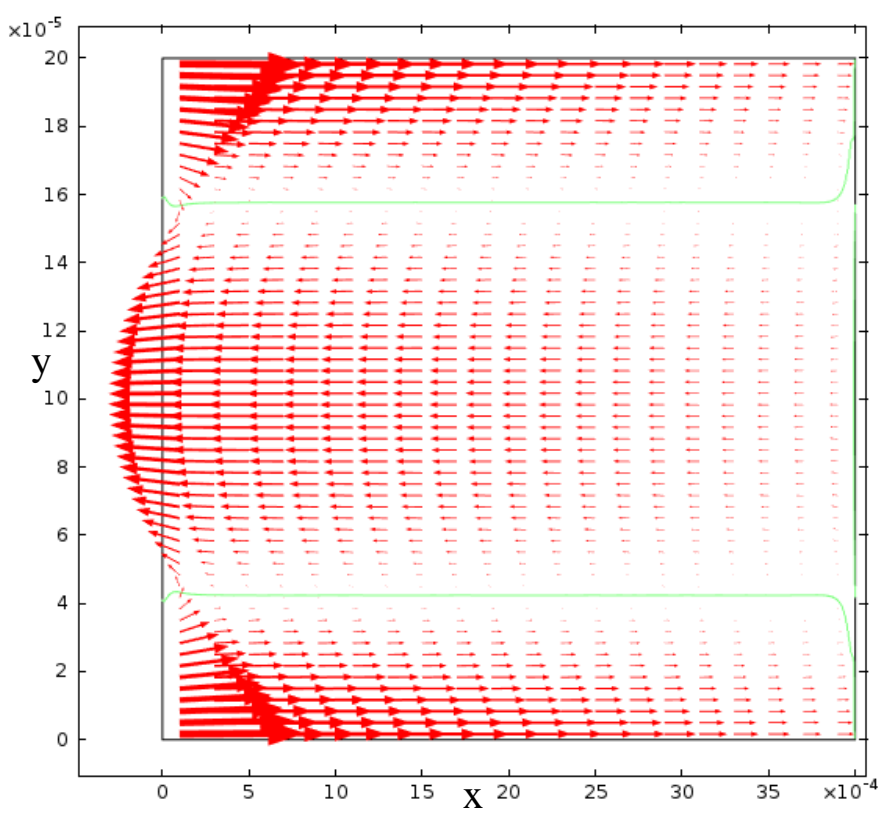

Figure 8. Velocity field within the channel at $100 \mathrm{~A} / \mathrm{m}^{2}$ current density; channel length $L=4$ $\mathrm{mm}$, half-height $h=100 \mu \mathrm{m}$, concentration ratio $C_{L} / C_{0}=100\left(u_{e o}>0\right)$. The green lines correspond to the positions of zero longitudinal velocity.

\subsection{Applicability of local Taylor-Aris approach}

As shown above, due to the transversal convection the Taylor-Aris approach is valid as a firstorder approximation only. As a quantitative criterion of accuracy of Taylor-Aris approximation we consider the deviation from unity of the ratio of solute flux, calculated numerically $\left(-D \frac{d \bar{C}}{d x}+\overline{u_{x} C}\right)$, to that given by the local Taylor-Aris approximation (Eq.(17))

$$
R=\frac{-D \frac{d \bar{C}}{d x}+\overline{u_{x} C}}{-D\left(1+\frac{2}{105} \frac{h^{2} u_{e o}^{2}}{D^{2}}\right) \frac{d \bar{C}}{d x}}=\frac{1}{1+\frac{2}{105} \frac{h^{2} u_{e o}^{2}}{D^{2}}}\left(1-\frac{\overline{u_{x} C}}{D \frac{d \bar{C}}{d x}}\right)
$$

One can relate this parameter with the correction $k$ to the Taylor-Aris model (Eqs. (24)-(25))

$$
R=1+\frac{k}{\frac{105 D^{2}}{2 h^{2} u_{e o}^{2}}+1}
$$

For large Peclet numbers one has approximately $R \approx 1+k$. In Figure 9 , profiles of parameter $R$ are shown for a channel of $4 \mathrm{~mm}$ length and $100 \mu \mathrm{m}$ half-height. The effect of current density $\left(100,200,300,400\right.$, and $\left.500 \mathrm{~A} / \mathrm{m}^{2}\right)$ is evaluated for the concentration ratio of 10 for two directions of electroosmotic flow: Figure 9a shows the case of electroosmosis directed from $x=$ 
0 to $x=L$, whereas Figure $9 \mathrm{~b}$ corresponds to the oppositely directed electroosmotic flow. Both cases show small deviations from the TAD theory in the inner part of the channel and strongly increasing deviations in narrow regions near the channel ends. Furthermore, at relatively low current densities, the deviations from TAD analysis are smaller and increasing with the current. Moreover, a clear dependence on the current direction is evident. The direction of current sets the value of correction $k$ (Eq.25) through the parameter $\chi$, defining the negative or positive deviation from TAD theory. On average, the absolute deviations are larger in Figure 9a.
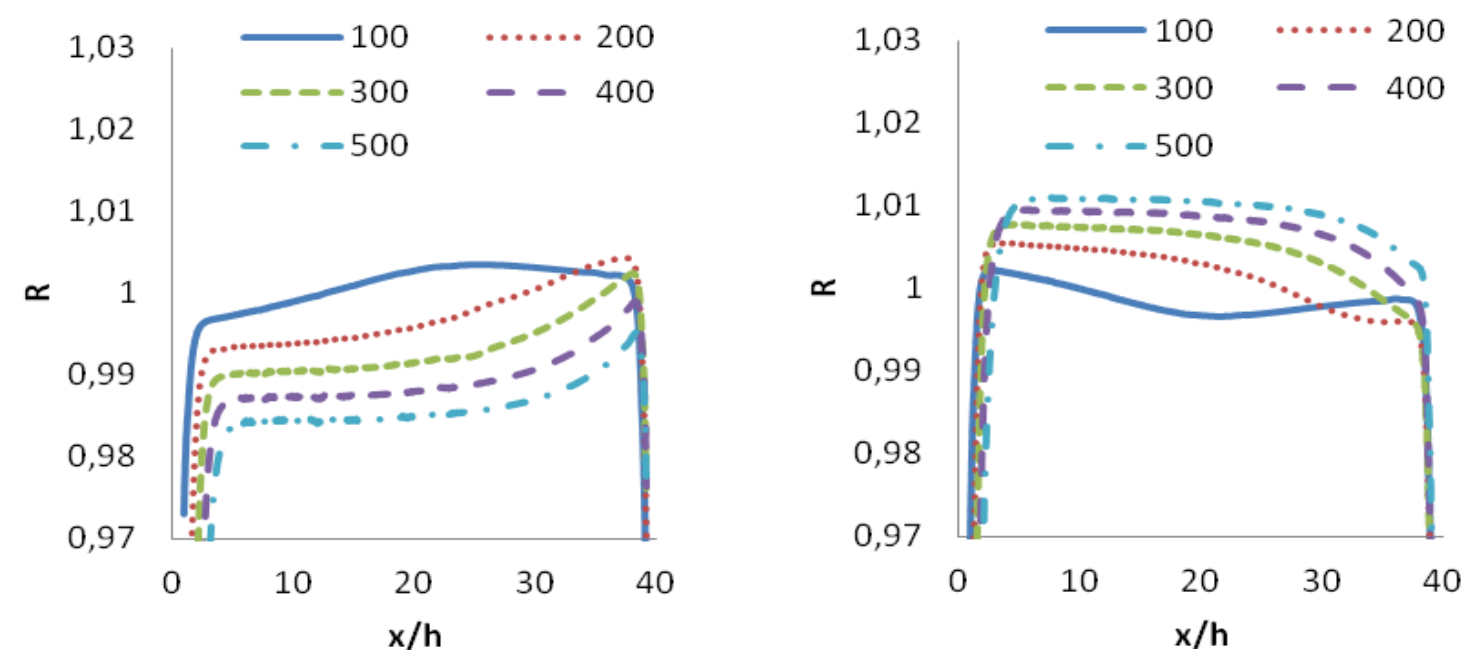

a)

b)

Figure 9. Deviations from the Taylor-Aris theory, quantified by parameter $R$, for the electroosmosis directed: a) from $x=0$ to $x=L$, and $\mathrm{b}$ ) in the opposite direction, along the channel of $4 \mathrm{~mm}$ length and half-height $h=100 \mu \mathrm{m}$ for various current densities $(100,200,300$, 400 , and $500 \mathrm{~A} / \mathrm{m}^{2}$ ) and the concentration ratio of 10 .

\subsection{Approximate parametric description}

As discussed above, near the channel ends boundary layers are formed where the concentration profiles transform from the constant concentrations at the channel boundaries $(x$ $=0, L)$ to those characteristic profiles of the TAD approximation at the internal edges of these boundary layers ( $x=x_{s}$ and $x=L-x_{m}$, see Fig.4). The origin of these boundary layers is qualitatively similar to the case of non-electrolyte solutions, considered in detail in Part I. The numerical simulations show that in those parts of the channel cross-section, where the fluid is entering the channel (e.g., at the channel walls), the strong convection makes the concentration practically equal to that at the boundary over some distance from it. The 
approximate parametric description, considered below, is based on the assumption that at the location where the wall concentration starts noticeably deviate from the boundary value the local Taylor-Aris approximation (with its characteristic transversal concentration profile) can already be used to calculate the concentration distribution within the inner part of the channel.

For the non-electrolyte transport problem (Part I), linear profiles of local concentration were obtained for the inner part of the channel. The length of boundary layers was defined via linear extrapolation of inner concentration profiles at the locations where the intrusion was most pronounced (for example, at the channel walls on the "entrance" side of the channel). In this study, due to the dependence of EOC rate on the local electrolyte concentration the concentration profiles are non-linear. This makes direct linear extrapolation impossible. However, above we have seen that at sufficiently large current densities linearity occurs for the profiles of reciprocal concentration (see Fig.4). Therefore, to define the length of intrusion zone, $x_{s}$, we can extrapolate the linear inner part of the profile of reciprocal local concentration at the walls (for the in-flowing solution) until it reaches the value $1 / C_{0}$ (see Figure 4) corresponding to the boundary condition. The normalized (by the channel half height, $h$ ) length of intrusion zone obtained in this way is presented in Table 1. Its length at the opposite end of the channel, $x_{m}$, can be obtained in a similar way. However, our numerical results show the intrusion zone at the channel edge with the higher concentration to be very short so we will assume $x_{m}=0$.

\begin{tabular}{|c|c|c|c|c|}
\hline & \multicolumn{4}{|c|}{$\boldsymbol{L} / \boldsymbol{h}$} \\
\hline$I\left(\mathrm{~A} / \mathrm{m}^{2}\right)$ & $\mathbf{1 0}$ & $\mathbf{2 0}$ & $\mathbf{4 0}$ & $\mathbf{6 0}$ \\
\hline 100 & 0.490 & 0.477 & 0.305 & 0.252 \\
\hline 200 & 0.863 & 0.697 & 0.508 & 0.421 \\
\hline 300 & 1.183 & 0.962 & 0.869 & 0.618 \\
\hline 400 & 1.554 & 1.290 & 1.151 & 0.980 \\
\hline 500 & 1.863 & 1.630 & 1.433 & 1.256 \\
\hline 600 & 2.130 & 1.934 & 1.715 & 1.499 \\
\hline 700 & 2.365 & 2.212 & 1.997 & 1.741 \\
\hline 800 & 2.580 & 2.469 & 2.279 & 1.974 \\
\hline
\end{tabular}

Table 1. Normalized length of intrusion zone, $x_{\S} / h$, for various current densities, $I$, and aspect ratios, $L / h$, and for the concentration ratio $C_{L} / C_{0}=10$.

Having estimated the length of intrusion zone, $x_{s}$, for various current densities, we can obtain the average concentrations at the internal sides of the boundary layers $C_{1}=\bar{C}\left(\tilde{x}_{s}\right)$ and 
$C_{2}=\bar{C}\left(1-\tilde{x}_{m}\right)$. These concentrations are determined as a solution of a set of two equations for two unknowns, $C_{1}$ and $C_{2}$ (Appendix D)

$$
C_{1}=C_{0}-\frac{F}{15\left(\frac{L}{h}-\frac{x_{S}}{h}\right)} \frac{\left[\left(C_{1}-C_{2}\right)+\frac{2 F^{2}}{105}\left(\frac{1}{C_{2}}-\frac{1}{C_{1}}\right)\right]}{C_{1}+\frac{2 F^{2}}{105} \frac{1}{C_{1}}}
$$

where the parameter $F=\frac{h \chi}{D}$ combines the physical properties of the system.

$$
C_{2}=C_{L}+\frac{7 F}{120\left(\frac{L}{h}-\frac{x_{S}}{h}\right)} \frac{\left[\left(C_{1}-C_{2}\right)+\frac{2 F^{2}}{105}\left(\frac{1}{C_{2}}-\frac{1}{C_{1}}\right)\right]}{C_{2}+\frac{2 F^{2}}{105} \frac{1}{C_{2}}}
$$

Once those concentrations are determined, they can be substituted to Eqs.(19) and (20) in order to obtain parametric equations for the description of average-concentration profile in the inner part of the channel by using the numerically obtained parameter $x_{s} / h$ (the parameter $x_{m} / h$ can be also accounted for)

$$
\begin{aligned}
& \bar{C}(x)=\frac{1}{2}\left(\tilde{Q}\left(1-\tilde{x}_{m}-\tilde{x}\right)+C_{2}-\frac{2 F^{2}}{105} \frac{1}{C_{2}}\right)+\sqrt{\frac{1}{4}\left(\tilde{Q}\left(1-\tilde{x}_{m}-\tilde{x}\right)+C_{2}-\frac{2 F^{2}}{105} \frac{1}{C_{2}}\right)^{2}+\frac{2 F^{2}}{105}} \\
& \tilde{Q}=\frac{1}{1-\tilde{x}_{S}-\tilde{x}_{m}}\left[\left(C_{1}-C_{2}\right)+\frac{2 F^{2}}{105}\left(\frac{1}{C_{2}}-\frac{1}{C_{1}}\right)\right]
\end{aligned}
$$

Figure 10 shows that the approximate values of concentration at the left-hand side of the channel, $C_{l}$, are slightly higher than $C_{0}$ whereas those at the right-hand side, $C_{2}$, are smaller than $C_{L}$. With increasing current density both the concentration "jumps", $C_{1}-C_{0}$ and $C_{L}-C_{2}$, are increasing. A good agreement is observed between the numerical and analytical results even for cases where $x_{m}$ was disregarded (Figure 10a). The agreement is better in the case of larger aspect ratios $(L / h)$ where the entrance zones make weaker contributions to deviation of the system behavior from that expected according to the TAD formalism. 


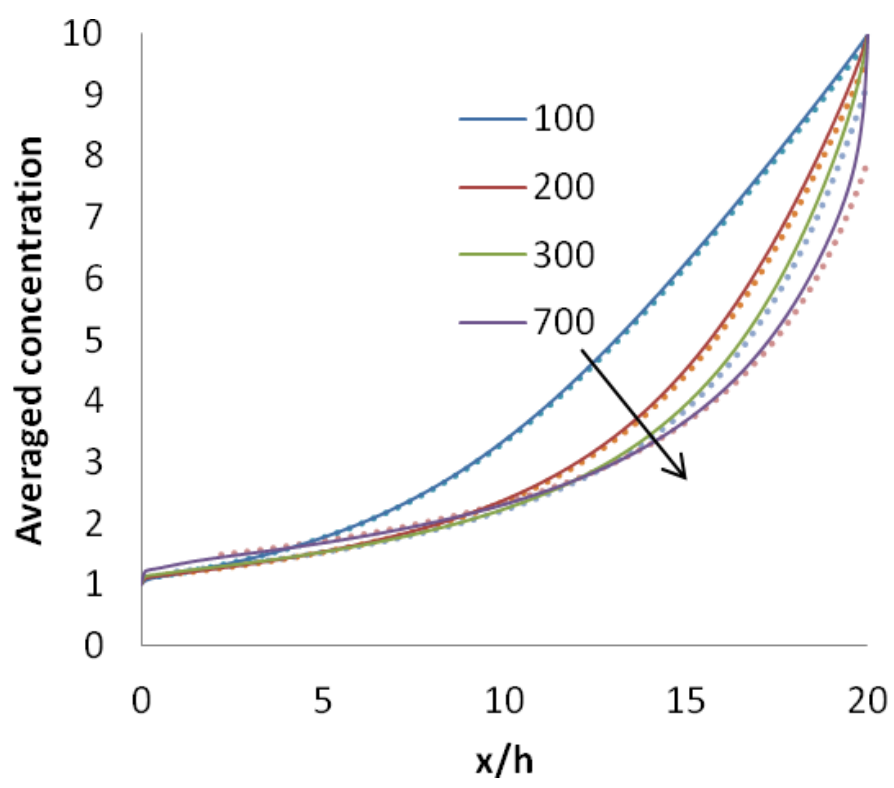

a)

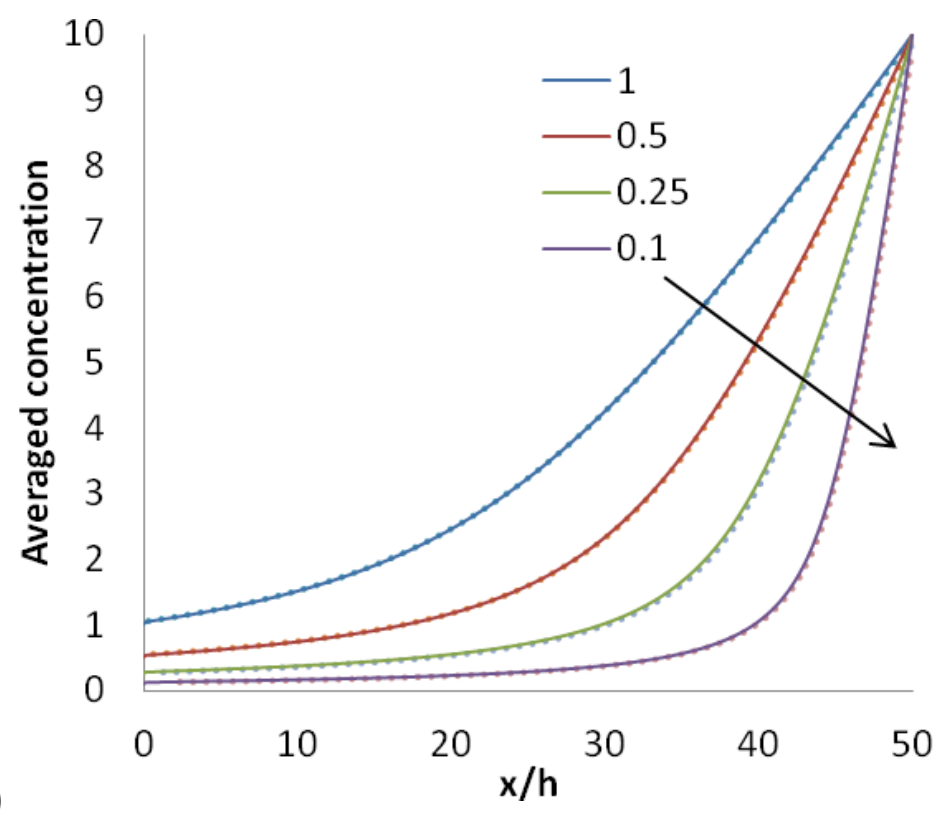

Figure 10. Comparison between the profiles of cross-section-averaged concentration calculated numerically and analytically (Eqs.(33)-(36)) for: a) aspect ratio $L / h=20$, concentration ratio $C_{L} / C_{0}=10$ and various current densities $\left(100,200,300\right.$ and $\left.700 \mathrm{~A} / \mathrm{m}^{2}\right) ;$ b) aspect ratio $L / h=50$, current density $100 \mathrm{~A} / \mathrm{m}^{2}$, and various values of the lower concentration $\left(C_{0}=1,0.5,0.25\right.$ and $0.1)$. 
Above we have seen that with increasing current density the salt-concentration profiles become ever more non-linear. Expectedly, this gives rise to non-linear current-voltage characteristics. The latter can be obtained by using this relationship:

$V=\frac{I}{\alpha} \cdot \int_{0}^{L} \frac{d x}{\bar{C}(x)}$

where $V$ is the voltage drop along the channel. Fig.11 shows some examples. The shape is surprisingly similar to that observed in the current-induced polarization of ion-exchange membranes. The current vs voltage curves have a characteristic shape with a quasi-plateau resembling a limiting current followed by a faster current increase ("over-limiting" current). The initial linear dependence changes into a gradually decreasing slope due to the fact that an ever larger part of the channel is occupied by the zone of lower concentration. This decrease in the slope practically stops when the profile of reciprocal concentration gets linear (see Fig.3). However, at still larger current densities, there is an increasing concentration "jump" at the channel entrance from the "dilute" side of the channel (see Fig.2). This leads to an increase of the local conductivity in the left part of the channel, i.e. in the part where it is the smallest, and an increase in the slope.

Fig.11 shows that for a rather broad range of currents (especially at larger aspect ratios) in terms of current-voltage characteristics there is a good agreement between the numerical results and the parameterization approach.

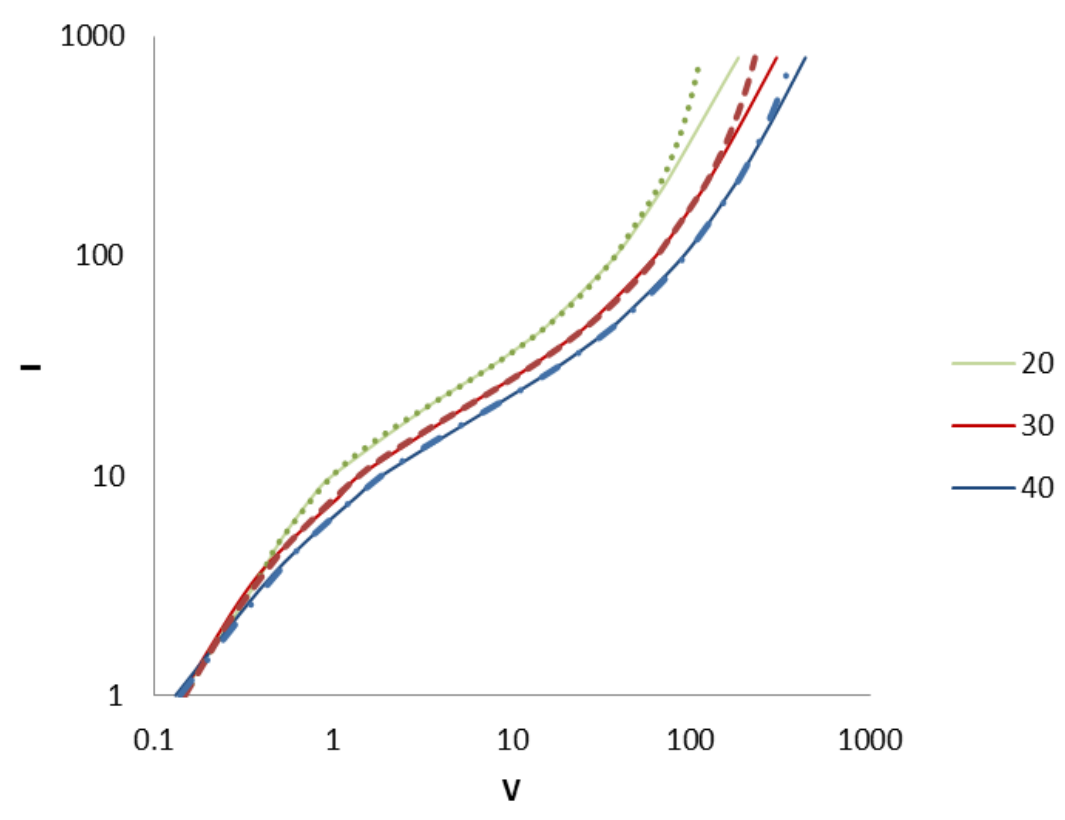


Figure 11. Current vs voltage curves for different aspect ratios $(L / h=20,30$ and 40$)$ with a concentration ratio $C_{L} / C_{0}=100$. Dashed lines correspond to the analytical procedure and solid lines were obtained numerically.

\subsection{Constant surface charge and constant electric potential as boundary condition at channel edges.}

An additional numerical simulation is presented in this section where instead of the case mentioned above with constant zeta potential equal to $0.1 \mathrm{~V}$ and constant current density at the edges of the channel (eq. 7), a constant surface charge model and constant potential at the channel edges was used. On one hand the boundary conditions at the edges are defined as:

$$
V(0, y)=V_{1} ; V(L, y)=0 ; \quad \boldsymbol{n} \cdot \boldsymbol{J}(x, \pm h)=0
$$

On the other hand, according to (Yaroshchuk 2011) for a case with strongly-acidic groups (constant-charge model) and for a Z:Z buffer electrolyte an analytical expression for zeta potential where it is express as function of a constant charge density and salt concentration can be defined as:

$\zeta=\frac{2}{z} \operatorname{asinh}\left(\frac{B}{2 \sqrt{R T s \varepsilon_{0} c}}\right)$

where $B$ is the surface charge density. Substituting eq. 39 in eq. 6 the electroosmotic velocity is expressed in terms of the surface charge. Figure 12 shows zeta potential as function of concentration with two values of surface charge density $\left(-5\right.$ and $\left.-30 \mathrm{mc} / \mathrm{m}^{2}\right)$ which are in a typical range of glass and silica surfaces at the concentrations we were considering with 1:1 electrolytes (Behrens and Grier 2001).

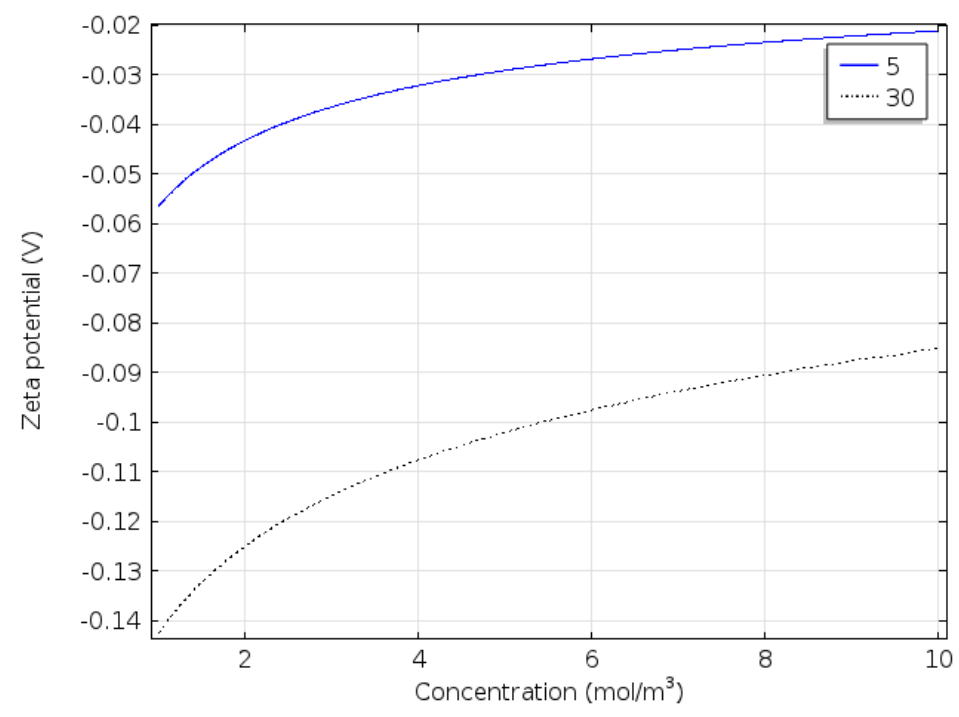

Figure 12. Zeta potential as function of concentration with two values of surface charge density -5 and $-30 \mathrm{mc} / \mathrm{m}^{2}$. 
As expected, lower values of surface charge produce lower values of zeta potential. Electroosmotic velocity is proportional to $\zeta$ whose dependence on the concentration create a stronger nonlinearity in the averaged concentration profile, including larger jumps at the diluted side of the channel as can be seen in Figure 13-a. Despite the evident differences between the two models, when comparing the resistance (eq. 37) of the most equivalent cases: that one with $-35 \mathrm{mC} / \mathrm{m}^{2}$ with $\mathrm{V}_{1}=35 \mathrm{~V}$ and the case of constant zeta potential and $300 \mathrm{~A} / \mathrm{m}^{2}$ of current density, its difference is only about 1.5\%. Furthermore, Figure 13-b shows a qualitatively similar behavior than that one observed in Figure 5 but with a larger region with disrupted profiles at the diluted side provoqued by the stronger dependance on concentration of the electroosmotic flow. Nevertheless, the existance of an inner region were the TAD theory is applicable is observed although smaller.

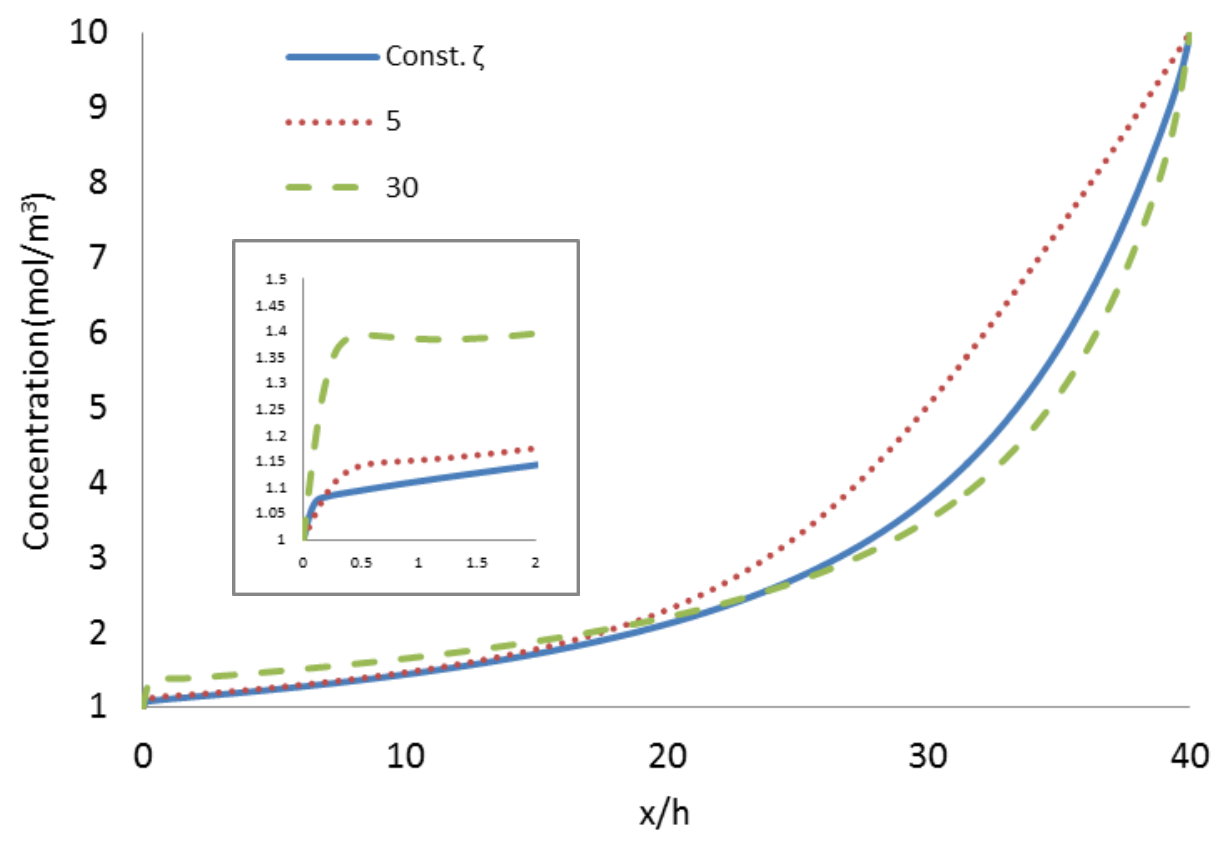

a) 


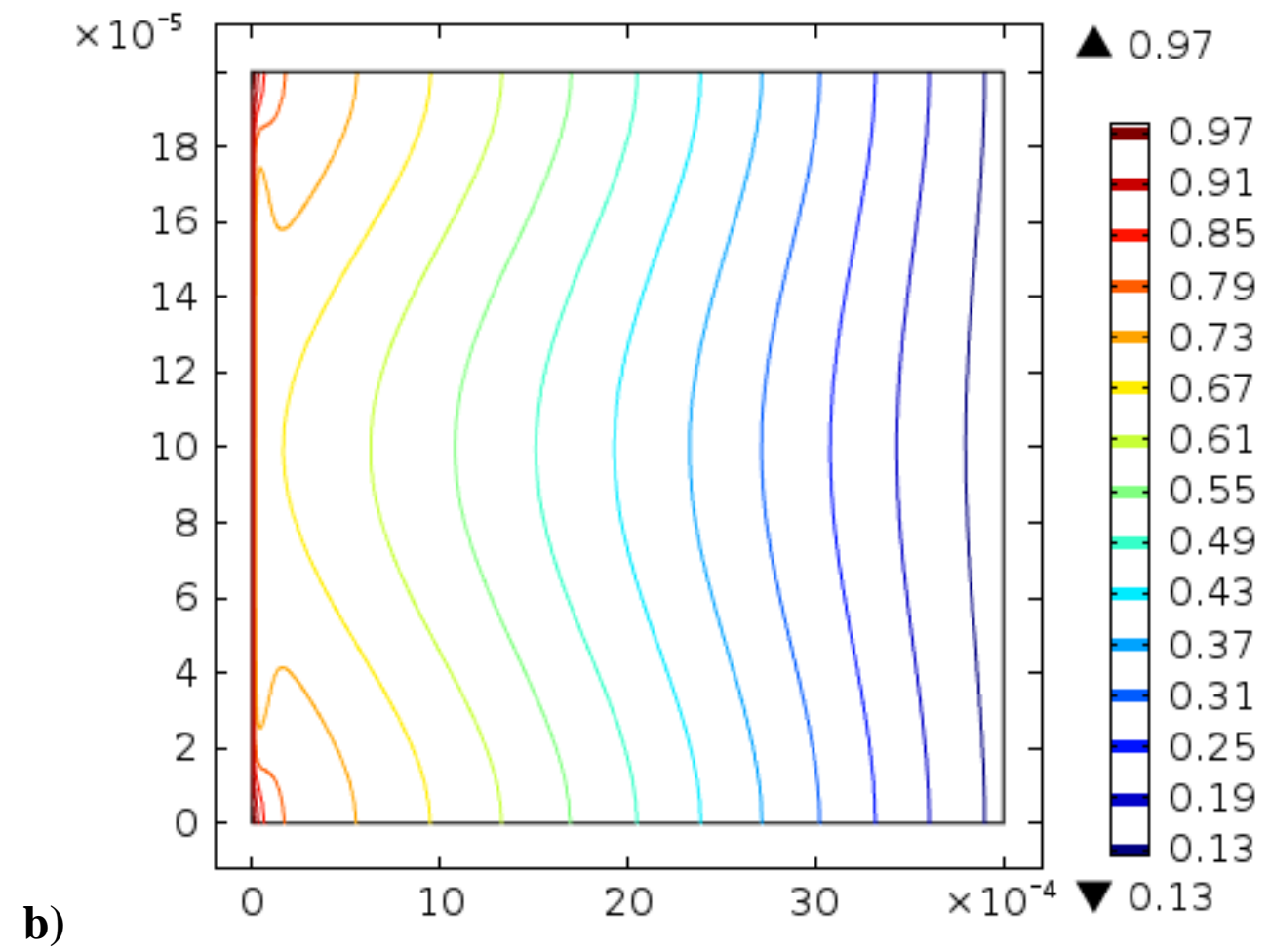

Figure 13. a)Comparison between the averaged concentration profiles in a $100 \mu \mathrm{m}$ height channel of $4 \mathrm{~mm}$ of length and constant zeta potential of $0.1 \mathrm{~V}$ under $300 \mathrm{~A} / \mathrm{m}^{2}$ of current density against the cases with variable zeta potential and surface charge equal to 5 and 30 $\mathrm{mC} / \mathrm{m}^{2}$ under a voltage difference of 35 volts. Inset represents a zoomed area near to the channel edge with low concentration. b) Contour graph showing the distribution of the reciprocal concentration within the channel at $35 \mathrm{v}$ of potential difference $\left(V_{1}\right)$, surface charge of $-35 \mathrm{mC} / \mathrm{m}^{2}$

\section{Conclusions}

The results of numerical simulations and approximate analytical solutions show that under conditions of electroosmotic circulation within a major part of a long channel the concentration distribution is in a good agreement with that predicted by the "local" TAD formalism. This fails only within two relatively narrow boundary layers formed at the channel edges. Due to the varying electroosmotic flow velocity along the channel the TAD approach should be used locally so the dispersion coefficient is related to the local Péclet number.

Due to the condition of constant concentrations imposed at the channel edges, the concentration profiles deviate from the Taylor-like shape within the boundary layers formed at the edges. This results in the appearance of "jumps" of average concentration close to the channel edges. The lengths of the entrance zones with distorted concentration profiles $\left(x_{s, m}\right)$ are controlled by the local concentration, electrical current density (electric field or local Péclet 
number) and the height of the channel. They are almost independent of the channel length until they increase to become comparable with it.

These entrance zones control the magnitude of "jumps" of average concentration at the channel edges and, thus, influence the inner concentration profile, too. With increasing $\mathrm{Pe}$, the external solutions from the reservoirs penetrate deeper into the channel and deform stronger the transversal concentration profiles, producing larger deviations from the TAD approximation in a larger portion of the channel. These features are confirmed by both numerical and analytical calculations. Minor deviations are observed between them in a rather wide range of electrical current densities, aspect ratios and concentration ratios.

An approximate parametric description can be proposed that simplifies the calculations of the concentration distribution within the channel and the current-voltage characteristics of the system. Such parametric description can be useful for the analysis of experimental data on the hydrodynamic dispersion under conditions of electroosmotic circulation and on the concentration polarization in long micro-channels terminated by ion-selective "plugs".

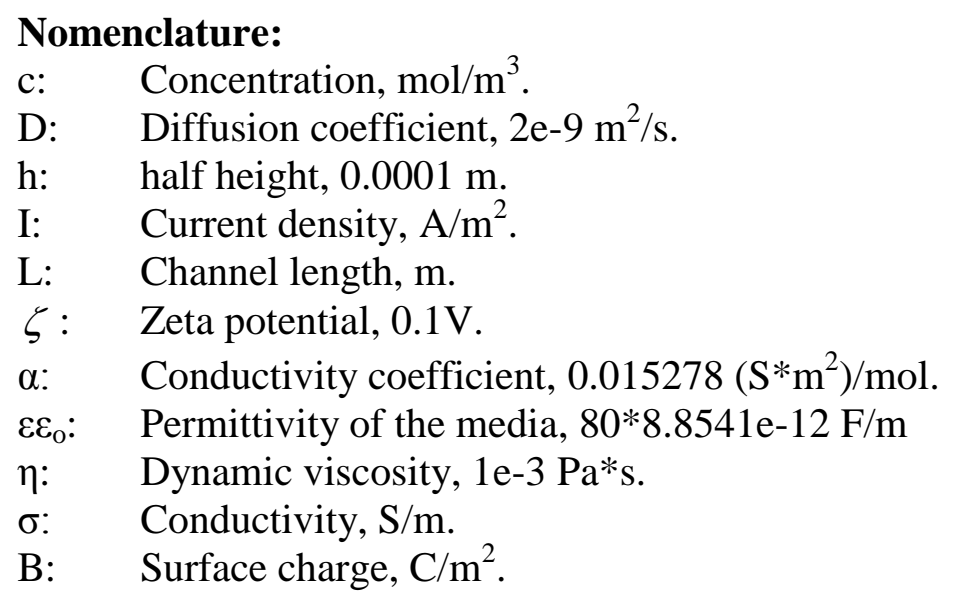

\section{Acknowledgements}

The work of EELB was supported by National Council of Science and Technology of Mexico (CONACYT) within the scope of fellowship reg./CVU: 213775/237269. Financial support from European Commission within the scope of FP7 (project acronym "CoTraPhen", Grant Agreement Number: PIRSES-GA-2010-269135) is gratefully acknowledged. 


\section{References}

Aris R (1956) On the dispersion of a solute in a fluid flowing through a tube. Proc R Soc London 235:67-77.

Behrens SH, Grier DG (2001) The charge of glass and silica surfaces. J Chem Phys 115:6716.

Deng D, Dydek EV, Han J-H, et al (2013) Overlimiting Current and Shock Electrodialysis in Porous Media. Langmuir 1-27. doi: 10.1021/la4040547

Dydek EV, Zaltzman B, Rubinstein I, et al (2011) Overlimiting Current in a Microchannel. Phys Rev Lett 107:118301. doi: 10.1103/PhysRevLett.107.118301

Kelly KC, Miller SA, Timperman AT (2009) Investigation of Zone Migration in a Current Rectifying Nanofluldic/Microfluidic Analyte Concentrator. Anal Chem 81:732-738. doi: $10.1021 / \mathrm{ac} 802209 \mathrm{e}$

Kim SJ, Wang Y-C, Lee JH, et al (2007) Concentration polarization and nonlinear electrokinetic flow near a nanofluidic channel. Phys Rev Lett 99:44501. doi:

10.1103/PhysRevLett.99.044501

Kovarik ML, Zhou K, Jacobson SC (2009) Effect of Conical Nanopore Diameter on Ion Current Rectification. J Phys Chem B 113:15960-15966. doi: 10.1021/jp9076189

Lee JSH, Ren CL, Li D (2005) Effects of surface heterogeneity on flow circulation in electroosmotic flow in microchannels. Anal Chim Acta 530:273-282. doi: 10.1016/j.aca.2004.09.026

Licon Bernal EE, Kovalchuk VI, Zholkovskiy EK, Yaroshchuk a. (2014a) Hydrodynamic dispersion in long microchannels under conditions of electroosmotic circulation. I. Nonelectrolytes. Microfluid Nanofluidics 1139-1154. doi: 10.1007/s10404-014-1506-8

Licon Bernal EE, Kovalchuk VI, Zholkovskiy EK, Yaroshchuk A (2014b) Hydrodynamic dispersion in long microchannels under conditions of electroosmotic circulation. I. Nonelectrolytes. Microfluid Nanofluidics. doi: 10.1007/s10404-014-1506-8

Mani A, Bazant MZ (2011) Deionization shocks in microstructures. Phys Rev E 84:61504. doi: 10.1103/PhysRevE.84.061504

Mani A, Zangle T a, Santiago JG (2009) On the propagation of concentration polarization from microchannel-nanochannel interfaces. Part I: Analytical model and characteristic analysis. Langmuir 25:3898-908. doi: 10.1021/la803317p

Panton RL (2013) Incompressible Flow. John Wiley \& Sons

Plecis A, Nanteuil C, Haghiri-Gosnet A-M, Chen Y (2008) Electropreconcentration with chargeselective nanochannels. Anal Chem 80:9542-50. doi: 10.1021/ac8017907

Postler T, Slouka Z, Svoboda M, et al (2008) Parametrical studies of electroosmotic transport characteristics in submicrometer channels. J Colloid Interface Sci 320:321-332. doi: 10.1016/j.jcis.2007.10.056 
Rubinstein I, Zaltzman B (2013) Convective diffusive mixing in concentration polarization: from Taylor dispersion to surface convection. J Fluid Mech 728:239-278. doi:

$10.1017 / \mathrm{jfm} .2013 .276$

Strickland DG, Suss ME, Zangle T a., Santiago JG (2010) Evidence shows concentration polarization and its propagation can be key factors determining electroosmotic pump performance. Sensors Actuators B Chem 143:795-798. doi: 10.1016/j.snb.2009.10.005

Suss ME, Mani A, Zangle TA, Santiago JG (2011) Electroosmotic pump performance is affected by concentration polarizations of both electrodes and pump. Sensors and Actuators APhysical 165:310-315. doi: 10.1016/j.sna.2010.10.002

Taylor GI (1953) Dispersion of soluble matter in solvent flowing slowly through a tube. Proc R Soc London 219:186-203.

Yaroshchuk A (2012) Over-limiting currents and deionization "shocks" in current-induced polarization: local-equilibrium analysis. Adv Colloid Interface Sci 183-184:68-81. doi: 10.1016/j.cis.2012.08.004

Yaroshchuk A, Zholkovskiy E, Pogodin S, Baulin V (2011) Coupled Concentration Polarization and Electroosmotic Circulation near Micro/Nanointerfaces: Taylor-Aris Model of Hydrodynamic Dispersion and Limits of Its Applicability. Langmuir 27:11710-11721. doi: $10.1021 / 1 \mathrm{a} 201354 \mathrm{~s}$

Yaroshchuk AE (2011) Transport properties of long straight nano-channels in electrolyte solutions: a systematic approach. Adv Colloid Interface Sci 168:278-91. doi: 10.1016/j.cis.2011.03.009

Zangle T a, Mani A, Santiago JG (2009) On the propagation of concentration polarization from microchannel-nanochannel interfaces. Part II: Numerical and experimental study. Langmuir 25:3909-16. doi: 10.1021/la803318e

Zangle T a, Mani A, Santiago JG (2010) Theory and experiments of concentration polarization and ion focusing at microchannel and nanochannel interfaces. Chem Soc Rev 39:1014-35. doi: 10.1039/b902074h

\section{Appendix A}

By using the dimensionless variables

$\tilde{x}=x / L, \quad \tilde{y}=y / h, \quad \tilde{u}_{x}=u_{x} / u_{C} \quad$ and $\quad \tilde{u}_{y}=L u_{y} / h u_{C}$

where $u_{c}$ is a characteristic velocity (which is chosen here as $u_{c}=u_{e o}(0)$ ), Eq.(12) can be represented in dimensionless form as

$\xi^{2}\left(-\frac{\partial^{2} \delta C}{\partial \tilde{x}^{2}}\right)+\xi P e\left(\overline{\tilde{u}}_{x} \frac{\partial \delta C}{\partial \tilde{x}}-\frac{d\left(\overline{\delta \tilde{u}_{x} \delta C}\right)}{d \tilde{x}}+\delta \tilde{u}_{x} \frac{d \bar{C}}{d \tilde{x}}+\delta \tilde{u}_{x} \frac{\partial \delta C}{\partial \tilde{x}}+\overline{\delta C \frac{\partial \delta \tilde{u}_{x}}{\partial \tilde{x}}}+\overline{\tilde{u}_{y}} \frac{\partial \delta C}{\partial \tilde{y}}+\delta \tilde{u}_{y} \frac{\partial \delta C}{\partial \tilde{y}}-\overline{\tilde{u}_{y}} \frac{\overline{\partial(\delta C)}}{\partial \tilde{y}}-\overline{\delta \tilde{u}_{y} \frac{\partial(\delta C)}{\partial \tilde{y}}}\right)-\frac{\partial^{2} \delta C}{\partial \tilde{y}^{2}}=0$ 
where $\xi=h / L$, and $P e=h u_{d} / D$ is the Péclet number. Representing the deviation of concentration from average as a series in powers of the small parameter $\xi$ (Eq.(13)) and neglecting the higher order terms in Eq.(A.2) one obtains

$\frac{\partial^{2} \delta C^{(0)}}{\partial \tilde{y}^{2}}=0$

With account for the conditions $\left.\frac{\partial \delta C}{\partial \tilde{y}}\right|_{y= \pm h}=0$ and $\delta \bar{C}=0$ this gives for the zero-order term in concentration $\delta C^{(0)}=0$. For the first-order term we have this equation

$\frac{\partial^{2} \delta C^{(1)}}{\partial \widetilde{y}^{2}}=P e\left(\overline{\tilde{u}}_{x} \frac{\partial \delta C^{(0)}}{\partial \tilde{x}}-\frac{d\left(\overline{\left.\delta \tilde{u}_{x} \delta C^{(0)}\right)}\right.}{d \tilde{x}}+\delta \tilde{u}_{x} \frac{d \bar{C}}{d \tilde{x}}+\delta \tilde{u}_{x} \frac{\partial \delta C^{(0)}}{\partial \tilde{x}}+\overline{\delta C^{(0)} \frac{\partial \delta \tilde{u}_{x}}{\partial \tilde{x}}}+\overline{\tilde{u}_{y}} \frac{\partial \delta C^{(0)}}{\partial \tilde{y}}+\delta \tilde{u}_{y} \frac{\partial \delta C^{(0)}}{\partial \tilde{y}}-\overline{\tilde{u}_{y}} \frac{\overline{\partial\left(\delta C^{(0)}\right)}}{\partial \tilde{y}}-\overline{\delta \tilde{u}_{y} \frac{\partial\left(\delta C^{(0)}\right)}{\partial \tilde{y}}}\right)(A$,

Substituting $\delta C^{(0)}=0$ one obtains

$\frac{\partial^{2} \delta C^{(1)}}{\partial \tilde{y}^{2}}=P e \cdot \delta \tilde{u}_{x} \cdot \frac{d \bar{C}}{d \tilde{x}}$

The last equation has the same form as in the case of constant electroosmotic velocity along the channel because the transversal velocity component contributes only to the higher order terms in the series for the deviation of concentration, Eq.(13). The solution of Eq.(A.5) is

$\delta C^{(1)} \approx-P e \cdot \omega \cdot \frac{d \bar{C}}{d \tilde{x}}$

where the function $\omega$ satisfy a simple differential equation

$\frac{\partial^{2} \omega}{\partial \tilde{y}^{2}}=-\tilde{u}_{x}$

with the boundary conditions: $\left.\frac{d \omega}{d y}\right|_{y=0, h}=0$ and $\bar{\omega}=0$. It is seen that the function $\omega$ is fully defined by the velocity distribution.

By substitution $\delta C \approx \xi \delta C^{(1)}$ with account for Eq.(A.6) the equation for the average concentration, Eq.(11), transforms into the following one

$\frac{d}{d \tilde{x}}\left[\left(1+P e^{2} \overline{\delta \tilde{u}_{x} \omega}\right) \frac{d \bar{C}}{d \tilde{x}}\right]-\frac{P e}{\xi}\left[\left(\tilde{u}_{x}-P e \xi \Omega(\tilde{x})\right)\right] \frac{d \bar{C}}{d \tilde{x}}=0$

where $\Omega(\tilde{x})=\delta \tilde{u}_{y}(\tilde{x}, 1) \omega(\tilde{x}, 1)-\delta \tilde{u}_{y}(\tilde{x},-1) \omega(\tilde{x},-1)+\overline{\tilde{u}}_{y}(\omega(\tilde{x}, 1)-\omega(\tilde{x},-1))$. For the considered here case of equally charged channel walls the velocity distribution within the channel is symmetrical. Under these conditions the function $\Omega(\tilde{x})$ turns to zero. We consider here also 
the case of a zero average longitudinal velocity, $\overline{\tilde{u}}_{x}=0$. Hence we have to omit the last term in Eq.(A.8) for our case to obtain Eq.(14).

For the second-order term Eq.(A.2) gives

$\frac{\partial^{2} \delta C^{(2)}}{\partial \tilde{y}^{2}}=P e\left(\overline{\tilde{u}}_{x} \frac{\partial \delta C^{(1)}}{\partial \tilde{x}}-\frac{d\left(\overline{\delta \tilde{u}_{x} \delta C^{(1)}}\right)}{d \tilde{x}}+\delta \tilde{u}_{x} \frac{\partial \delta C^{(1)}}{\partial \tilde{x}}+\overline{\delta C^{(1)} \frac{\partial \delta \tilde{u}_{x}}{\partial \tilde{x}}}+\overline{\tilde{u}}_{y} \frac{\partial \delta C^{(1)}}{\partial \tilde{y}}+\delta \tilde{u}_{y} \frac{\partial \delta C^{(1)}}{\partial \tilde{y}}-\overline{\tilde{u}_{y}} \frac{\overline{\partial\left(\delta C^{(1)}\right)}}{\partial \tilde{y}}-\delta \tilde{u}_{y} \frac{\partial\left(\delta C^{(1)}\right)}{\partial \tilde{y}}\right)$

Substituting $u_{x}, u_{y}$ and $\delta C^{(1)}$, given by Eqs.(15), (21) and (22), one obtains

$\frac{16}{P e^{2}} \frac{\partial^{2} \delta C^{(2)}}{\partial \tilde{y}^{2}}=\tilde{u}_{e o} \frac{d \tilde{u}_{e o}}{d \tilde{x}} \frac{d \bar{C}}{d \tilde{x}}\left(\frac{1}{7}-\frac{3 \tilde{y}^{2}}{5}+\tilde{y}^{4}-\tilde{y}^{6}\right)+\tilde{u}_{e o}^{2} \frac{d^{2} \bar{C}}{d \tilde{x}^{2}}\left(-\frac{17}{105}+\frac{17 \tilde{y}^{2}}{5}-7 \tilde{y}^{4}+3 \tilde{y}^{6}\right)$

Integration of this equation with the additional conditions $\left.\frac{\partial \delta C^{(2)}}{\partial \tilde{y}}\right|_{\tilde{y}=0}=0$ and $\overline{\delta C^{(2)}}=0$ yields

$\frac{16}{P e^{2}} \delta C^{(2)}=\tilde{u}_{e o} \frac{d \tilde{u}_{e o}}{d \tilde{x}} \frac{d \bar{C}}{d \tilde{x}}\left(-\frac{209}{12600}+\frac{\tilde{y}^{2}}{14}-\frac{\tilde{y}^{4}}{20}+\frac{\tilde{y}^{6}}{30}-\frac{\tilde{y}^{8}}{56}\right)+\tilde{u}_{e o}^{2} \frac{d^{2} \bar{C}}{d \tilde{x}^{2}}\left(-\frac{29}{12600}-\frac{17 \tilde{y}^{2}}{210}+\frac{17 \tilde{y}^{4}}{60}-\frac{7 \tilde{y}^{6}}{30}+\frac{3 \tilde{y}^{8}}{56}\right)$

With this result and accounting for Eqs.(13) and (21) one finds

$\frac{d\left(\overline{\delta u_{x} \delta C}\right)}{d x}=\frac{u_{C}}{L} \frac{d}{d \tilde{x}}\left(\xi \overline{\delta \tilde{u}_{x} \delta C^{(1)}}+\xi^{2} \overline{\delta \tilde{u}_{x} \delta C^{(2)}}\right)=\frac{u_{C}}{L} \frac{d}{d \tilde{x}}\left(-\xi \frac{2 P e \tilde{u}_{e o}^{2}}{105} \frac{d \bar{C}}{d \tilde{x}}+\xi^{2} \frac{2 P e^{2} \tilde{u}_{e o}^{2}}{105 \cdot 55} \frac{d \tilde{u}_{e o}}{d \tilde{x}} \frac{d \bar{C}}{d \tilde{x}}++\xi^{2} \frac{4 P e^{2} \widetilde{u}_{e o}^{3}}{105 \cdot 165} \frac{d^{2} \bar{C}}{d \tilde{x}^{2}}\right)$

Then Eq.(11) for the average concentration transforms to Eq.(23).

\section{Appendix B}

Because of the presence of transversal velocity component the velocity distribution given by Eqs.(15) and (22) is an approximation valid for sufficiently long narrow channels. Let us consider the full set of steady-state Navier-Stokes and continuity equations in a dimensionless form

$$
\begin{aligned}
& \xi \operatorname{Re}\left(\tilde{u}_{x} \frac{\partial \tilde{u}_{x}}{\partial \tilde{x}}+\tilde{u}_{y} \frac{\partial \tilde{u}_{x}}{\partial \tilde{y}}\right)=-\frac{\partial \tilde{P}}{\partial \tilde{x}}+\xi^{2} \frac{\partial^{2} \tilde{u}_{x}}{\partial \tilde{x}^{2}}+\frac{\partial^{2} \tilde{u}_{x}}{\partial \tilde{y}^{2}} \\
& \xi \operatorname{Re}\left(\tilde{u}_{x} \frac{\partial \tilde{u}_{y}}{\partial \tilde{x}}+\tilde{u}_{y} \frac{\partial \tilde{u}_{y}}{\partial \tilde{y}}\right)=-\frac{1}{\xi^{2}} \frac{\partial \tilde{P}}{\partial \tilde{y}}+\xi^{2} \frac{\partial^{2} \tilde{u}_{y}}{\partial \tilde{x}^{2}}+\frac{\partial^{2} \tilde{u}_{y}}{\partial \tilde{y}^{2}} \\
& \frac{\partial \tilde{u}_{y}}{\partial \tilde{x}}+\frac{\partial \tilde{u}_{y}}{\partial \tilde{y}}=0
\end{aligned}
$$

where the dimensionless variables are defined as $\tilde{x}=x / L, \tilde{y}=y / h, \quad \tilde{u}_{x}=u_{x} / u_{C}$, $\tilde{u}_{y}=L u_{y} / h u_{C}, \xi=h / L, \tilde{P}=h^{2} P / \rho v u_{C} L, P$ is the pressure, $\rho$ is the solution density, $v$ is the kinematic viscosity, and $R e=h u_{C} / v$ is the Reynolds number. 
Representing the velocity components and the pressure as series in powers of the small parameter $\xi$, similar to Eq.(13),

$\tilde{u}_{x}=\tilde{u}_{x}^{(0)}+\xi \tilde{u}_{x}^{(1)}+\xi^{2} \tilde{u}_{x}^{(2)}+\ldots$

$\tilde{u}_{y}=\tilde{u}_{y}^{(0)}+\xi \tilde{u}_{y}^{(1)}+\xi^{2} \tilde{u}_{y}^{(2)}+\ldots$

$\widetilde{P}=\tilde{P}^{(0)}+\xi \tilde{P}^{(1)}+\xi^{2} \tilde{P}^{(2)}+\ldots$

one obtains from Eq.(B.2) as a zero order approximation $\frac{\partial \widetilde{P}^{(0)}}{\partial \tilde{y}}=0$, what means that in a long channel the pressure is a function of the coordinate $\mathrm{x}$ only: $\widetilde{P}^{(0)}=\widetilde{P}^{(0)}(\tilde{x})$. From Eq.(B.1) one obtains

$\frac{d \widetilde{P}^{(0)}}{d \widetilde{x}}=\frac{\partial^{2} \tilde{u}_{x}^{(0)}}{\partial \tilde{y}^{2}}$

Because the pressure is independent of the coordinate $y$, we can integrate this equation with the boundary conditions $\tilde{u}_{x}^{(0)}(\tilde{x}, 1)=\tilde{u}_{e o}(\tilde{x})$ and $\left(\frac{d \tilde{u}_{x}^{(0)}}{d \tilde{y}}\right)_{\tilde{y}=0}=0$ to obtain

$\tilde{u}_{x}^{(0)}=\tilde{u}_{e o}-\frac{d \tilde{P}^{(0)}}{d \tilde{x}}\left(\frac{1}{2}-\frac{\tilde{y}^{2}}{2}\right)$

The full volume flux through the channel, $\Phi$, is a constant

$\Phi=\int_{-1}^{1} \tilde{u}_{x}^{(0)} d \tilde{y}=2 \tilde{u}_{e o}-\frac{2}{3} \frac{d \tilde{P}^{(0)}}{d \tilde{x}}$

therefore the pressure drop can be expressed through this volume flux and Eq.(B.8) turns to

$\tilde{u}_{x}^{(0)}=\tilde{u}_{e o}\left(-\frac{1}{2}+\frac{3 \tilde{y}^{2}}{2}\right)+\frac{3}{4} \Phi\left(1-\tilde{y}^{2}\right)$

For $\Phi=0$ this equation transforms to Eq.(15). For small electroosmotic velocities, satisfying the condition $\operatorname{Re} \ll h / L$, one finds: $\tilde{u}_{x}^{(1)}=0, \quad \tilde{u}_{y}^{(1)}=0$, and $\tilde{P}^{(1)}=0$. For the second-order approximation one has from Eqs.(B.1) and (B.2)

$$
\begin{aligned}
& \frac{\partial \widetilde{P}^{(2)}}{\partial \tilde{x}}=\frac{\partial^{2} \tilde{u}_{x}^{(0)}}{\partial \tilde{x}^{2}}+\frac{\partial^{2} \tilde{u}_{x}^{(2)}}{\partial \tilde{y}^{2}} \\
& \frac{\partial \widetilde{P}^{(2)}}{\partial \tilde{y}}=\frac{\partial^{2} \tilde{u}_{y}^{(0)}}{\partial \tilde{y}^{2}}
\end{aligned}
$$

what gives (for $\Phi=0$ )

$$
\tilde{u}_{x}^{(2)}=-\frac{d^{2} \tilde{u}_{e o}}{d \tilde{x}^{2}}\left(\frac{1}{20}-\frac{3 \tilde{y}^{2}}{10}+\frac{\tilde{y}^{4}}{4}\right)
$$




$$
\begin{aligned}
& \tilde{u}_{y}^{(2)}=-\frac{d^{3} \widetilde{u}_{e o}}{d \tilde{x}^{3}} \frac{\tilde{y}\left(1-\tilde{y}^{2}\right)^{2}}{20} \\
& \widetilde{P}^{(2)}=\frac{d \widetilde{u}_{e o}}{d \tilde{x}}\left(\frac{1}{10}-\frac{3 \tilde{y}^{2}}{2}\right)
\end{aligned}
$$

With account for the contribution of the second-order terms for the velocity Eq.(24) preserves its form, but with the parameter $k$ including an additional term

$$
k=-\xi \frac{P e}{55} \frac{d \tilde{u}_{e o}}{d \tilde{x}}-\xi \frac{2 P e \tilde{u}_{e o}}{165} \frac{d^{2} \bar{C}}{d \tilde{x}^{2}}\left(\frac{d \bar{C}}{d \tilde{x}}\right)^{-1}+\xi^{2} \frac{1}{15 \tilde{u}_{e o}} \frac{d^{2} \tilde{u}_{e o}}{d \tilde{x}^{2}}
$$

It can be shown that this addition term can be neglected for sufficiently long channels, provided that $\frac{u_{e o} L}{D}>>\frac{22}{3} \frac{(C(L)-C(0))}{C(0)}$.

For another limiting case, $\operatorname{Re}>>h / L$, the equations for the first-order terms take the form

$$
\begin{aligned}
& \frac{\partial^{2} \tilde{u}_{x}^{(1)}}{\partial \tilde{y}^{2}}=\frac{\partial \widetilde{P}^{(1)}}{\partial \tilde{x}}+\operatorname{Re}\left(\tilde{u}_{x}^{(0)} \frac{\partial \tilde{u}_{x}^{(0)}}{\partial \tilde{x}}+\tilde{u}_{y}^{(0)} \frac{\partial \tilde{u}_{x}^{(0)}}{\partial \tilde{y}}\right) \\
& \frac{\partial \tilde{P}^{(1)}}{\partial \tilde{y}}=0
\end{aligned}
$$

Their solution for $\Phi=0$ gives the first-order terms

$$
\begin{aligned}
& \tilde{u}_{x}^{(1)}=\frac{\operatorname{Re} \tilde{u}_{e o}}{20} \frac{d \tilde{u}_{e o}}{d \tilde{x}}\left(\frac{1}{7}-\frac{9 \tilde{y}^{2}}{14}+\frac{\tilde{y}^{6}}{2}\right) \\
& \tilde{u}_{y}^{(1)}=-\frac{\operatorname{Re}}{280}\left(\tilde{u}_{e o} \frac{d^{2} \tilde{u}_{e o}}{d \tilde{x}^{2}}+\left(\frac{d \tilde{u}_{e o}}{d \tilde{x}}\right)^{2}\right)\left(2 \tilde{y}-3 \tilde{y}^{3}+\tilde{y}^{7}\right) \\
& \frac{d \tilde{P}^{(1)}}{d \tilde{x}}=-\frac{33 \operatorname{Re} \tilde{u}_{e o}}{105} \frac{d \tilde{u}_{e o}}{d \tilde{x}}
\end{aligned}
$$

With their account Eq.(24) remains the same again, but the parameter $k$ includes now an additional term

$$
k=-\xi \frac{P e}{55} \frac{d \tilde{u}_{e o}}{d \tilde{x}}-\xi \frac{2 P e \tilde{u}_{e o}}{165} \frac{d^{2} \bar{C}}{d \tilde{x}^{2}}\left(\frac{d \bar{C}}{d \tilde{x}}\right)^{-1}-\xi \frac{105 R e}{9702} \frac{d u_{e o}}{d \tilde{x}}
$$

The contribution of this addition term is negligibly small, because of $\mathrm{Re} \ll \mathrm{Pe}$.

\section{Appendix C}

To demonstrate that, within the frameworks of the employed approximation, the transverse electric filed can be ignored we consider a boundary value problem within a slit channel. The problem contains continuity equation for migration electric current in $\mathrm{KCl}$ (equal ionic 
mobilities) electrolyte solution having a given spatial distribution of concentration, $C(x, y)$. Accounting for the migration component of electric current, only, yields

$\nabla \cdot(\mathbf{E} C)=0$

The above equation is subject to boundary conditions imposing zero normal electric current density at the channel walls and constant electric potentials within the cross-sections at the channel ends. The difference between these potentials is set to be $U$.

$\mathbf{E}(x, \pm h) \cdot \mathbf{i}_{y}=0$

$\mathbf{E}(0, y) \cdot \mathbf{i}_{y}=0 ; \mathbf{E}(L, y) \cdot \mathbf{i}_{y}=0$

$\int_{0}^{L} \mathbf{E}(x, y) \cdot \mathbf{i}_{x} d x=U$

We will analyze the problem given by Eqs.(C.1) and (C.2) by following the next steps:

(i) Assume that the transverse electric field strength is zero within the whole channel, i.e., $\mathbf{E}=E_{x}(x, y) \cdot \mathbf{i}_{x}$.

(ii) Determine the function $E_{x}(x, y)$ by substituting the above form of $\mathbf{E}$ in Eqs. (C.1) and (C.2) and solving the obtained scalar problem.

(iii) Determine a posteriori $E_{y}(x, y)$ with the help of the relationship: $\partial E_{x} / \partial y=\partial E_{y} / \partial x$ which follows from the potentiality of electric field, $\nabla \times \mathbf{E}=0$.

By making steps ( $\mathrm{i}$ ) and (ii) we arrive at the following expression:

$$
E_{x}(x, y)=\frac{U}{C(x, y) \int_{0}^{L} 1 / C(x, y) d x}
$$

Determining $E_{y}(x, y)$, according to step (iii), leads to the following result

$$
E_{y}(x, y)=U \frac{\partial}{\partial y}\left[\frac{\int_{0}^{x} \frac{d x^{\prime}}{C\left(x^{\prime}, y\right)}}{\int_{0}^{L} \frac{d x}{C(x, y)}}\right]
$$

Now, we combine Eqs.(18), (10) and (21) to represent the obtained concentration distributions in the form

$$
C(x, y)=\bar{C}(x)\left[1+\frac{\xi \cdot P e \cdot \chi}{u_{e o}(0)}\left(-\frac{7}{120}+\frac{\tilde{y}^{2}}{4}-\frac{\tilde{y}^{4}}{8}\right) \cdot \frac{d}{d \tilde{x}}\left(\frac{1}{\bar{C}(x)}\right)\right]
$$

For the most interesting case of high Peclet numbers, by using Eq.(29), Eq. (C.5) can be represented as asymptotic form

$C(x, y)=\bar{C}(x) f(y)$ 
$f(y)=1+\frac{\xi \cdot P e \cdot \chi}{u_{e o}(0)}\left(-\frac{7}{120}+\frac{\tilde{y}^{2}}{4}-\frac{\tilde{y}^{4}}{8}\right) \cdot\left(\frac{1}{\bar{C}(L)}-\frac{1}{\bar{C}(0)}\right)$

Substituting Eq.(C.6) into Eq.(C.4) leads to conclusion $E_{y}(x, y)=0$. As well, substituting Eq.(C.6) into Eq.(C.3) shows that the longitudinal electric-field component, $E_{x}$, is independent of the transversal coordinate for this particular concentration distribution.

\section{Appendix D}

According to Eq.(21) we have for $x=x_{s}$ and $x=L-x_{m}$

$$
\begin{aligned}
& \bar{C}\left(\tilde{x}_{S}\right)=C_{0}-\delta C\left(\tilde{x}_{S}, 1\right)=C_{0}+\frac{\xi \cdot P e}{15} \cdot\left(\tilde{u}_{e o}(\tilde{x}) \cdot \frac{d \bar{C}}{d \tilde{x}}\right)_{\tilde{x}=\tilde{x}_{S}} \\
& \bar{C}\left(1-\tilde{x}_{m}\right)=C_{L}-\delta C\left(1-\tilde{x}_{m}, 0\right)=C_{L}-\frac{7 \xi \cdot P e}{120} \cdot\left(\tilde{u}_{e o}(\tilde{x}) \cdot \frac{d \bar{C}}{d \tilde{x}}\right)_{\tilde{x}=1-\tilde{x}_{m}}
\end{aligned}
$$

From Eq.(17) we have

$$
\frac{d \bar{C}}{d \tilde{x}}=-\frac{Q L}{D\left(1+\frac{2 P e^{2} \tilde{u}_{e o}^{2}}{105}\right)}
$$

Substituting $\frac{d \bar{C}}{d \tilde{x}}$ to Eqs. (D.1) and (D.2) we obtain

$$
\begin{aligned}
& \bar{C}\left(\tilde{x}_{S}\right)=C_{0}-\frac{\xi \cdot P e}{15} \cdot \frac{Q L}{D}\left(\frac{\tilde{u}_{e o}(\tilde{x})}{\left.1+\frac{2 P e^{2} \tilde{u}_{e o}^{2}}{105}\right)_{\tilde{x}=\tilde{x}_{S}}}\right. \\
& \bar{C}\left(1-\tilde{x}_{m}\right)=C_{L}+\frac{7 \xi \cdot P e}{120} \cdot \frac{Q L}{D}\left(\frac{\tilde{u}_{e o}(\tilde{x})}{1+\frac{2 P e^{2} \tilde{u}_{e o}^{2}}{105}}\right)_{\tilde{x}=1-\tilde{x}_{m}}
\end{aligned}
$$

The total flux $Q$ is obtained by modifying Eq.(20)

$$
\frac{Q L}{D}=-\frac{1}{1-\tilde{x}_{S}-\tilde{x}_{m}}\left(\bar{C}\left(1-\tilde{x}_{m}\right)-\bar{C}\left(\tilde{x}_{S}\right)\right)+\frac{2 P e^{2}}{105} \frac{\bar{C}^{2}\left(\tilde{x}_{S}\right)}{1-\tilde{x}_{S}-\tilde{x}_{m}}\left(\frac{1}{\bar{C}\left(1-\tilde{x}_{m}\right)}-\frac{1}{\bar{C}\left(\tilde{x}_{S}\right)}\right)
$$

Substituting $\tilde{u}_{e o}(\tilde{x})=\frac{u_{e o}(\tilde{x})}{u_{c}} \approx \frac{\chi}{u_{c} \bar{C}(\tilde{x})}$ to Eqs. (D.4) and (D.5) we obtain

$$
\bar{C}\left(\tilde{x}_{S}\right)=C_{0}-\frac{\xi \cdot P e}{15} \cdot \frac{Q L}{D}\left(\frac{\frac{\chi}{u_{c} \bar{C}(\tilde{x})}}{1+\frac{2 P e^{2}}{105} \frac{\chi^{2}}{u_{c}^{2} \bar{C}^{2}(\tilde{x})}}\right)_{\tilde{x}=\tilde{x}_{S}}
$$


$\bar{C}\left(1-\tilde{x}_{m}\right)=C_{L}+\frac{7 \xi \cdot P e}{120} \cdot \frac{Q L}{D}\left(\frac{\frac{\chi}{u_{c} \bar{C}(\tilde{x})}}{1+\frac{2 P e^{2}}{105} \frac{\chi^{2}}{u_{c}^{2} \bar{C}^{2}(\tilde{x})}}\right)_{\tilde{x}=1-\tilde{x}_{m}}$

Then using $P e=\frac{h u_{c}}{D}=\frac{h u_{e o}\left(\tilde{x}_{s}\right)}{D}=\frac{h \chi}{D \bar{C}\left(\tilde{x}_{s}\right)}$ and $\tilde{Q}=\frac{Q L}{D}$ we find

$$
\bar{C}\left(\tilde{x}_{S}\right)=C_{0}-\frac{\xi \cdot h \chi \tilde{Q}}{15 D} \frac{1}{\bar{C}\left(\tilde{x}_{S}\right)+\frac{2 h^{2} \chi^{2}}{105 D^{2}} \frac{1}{\bar{C}\left(\tilde{x}_{S}\right)}}
$$

$\bar{C}\left(1-\tilde{x}_{m}\right)=C_{L}+\frac{7 \xi \cdot h \chi \tilde{Q}}{120 D} \cdot \frac{1}{\bar{C}\left(1-\tilde{x}_{m}\right)+\frac{2 h^{2} \chi^{2}}{105 D^{2}} \frac{1}{\bar{C}\left(1-\tilde{x}_{m}\right)}}$

$\tilde{Q}=-\frac{1}{1-\tilde{x}_{S}-\tilde{x}_{m}}\left(\bar{C}\left(1-\tilde{x}_{m}\right)-\bar{C}\left(\tilde{x}_{S}\right)\right)+\frac{2 h^{2} \chi^{2}}{105 D^{2}} \frac{1}{1-\tilde{x}_{S}-\tilde{x}_{m}}\left(\frac{1}{\bar{C}\left(1-\tilde{x}_{m}\right)}-\frac{1}{\bar{C}\left(\tilde{x}_{S}\right)}\right)$

Substituting $C_{1}=\bar{C}\left(\tilde{x}_{s}\right), C_{2}=\bar{C}\left(1-\tilde{x}_{m}\right)$ and $F=\frac{h \chi}{D}$

$C_{1}=C_{0}-\frac{\xi \cdot F \tilde{Q}}{15} \frac{1}{C_{1}+\frac{2 F^{2}}{105} \frac{1}{C_{1}}}$

$C_{2}=C_{L}+\frac{7 \xi \cdot F \tilde{Q}}{120} \cdot \frac{1}{C_{2}+\frac{2 F^{2}}{105} \frac{1}{C_{2}}}$

$\tilde{Q}=\frac{1}{1-\tilde{x}_{S}-\tilde{x}_{m}}\left[\left(C_{1}-C_{2}\right)+\frac{2 F^{2}}{105}\left(\frac{1}{C_{2}}-\frac{1}{C_{1}}\right)\right]$

Finally, substituting Eq.(D.14) to (D.12) and (D.13) and neglecting $x_{m}$ we obtain

$$
\begin{gathered}
C_{1}=C_{0}-\frac{\xi \cdot F}{15\left(1-\tilde{x}_{S}\right)} \frac{\left[\left(C_{1}-C_{2}\right)+\frac{2 F^{2}}{105}\left(\frac{1}{C_{2}}-\frac{1}{C_{1}}\right)\right]}{C_{1}+\frac{2 F^{2}}{105} \frac{1}{C_{1}}} \\
C_{2}=C_{L}+\frac{7 \xi \cdot F}{120\left(1-\tilde{x}_{S}\right)} \frac{\left[\left(C_{1}-C_{2}\right)+\frac{2 F^{2}}{105}\left(\frac{1}{C_{2}}-\frac{1}{C_{1}}\right)\right]}{C_{2}+\frac{2 F^{2}}{105} \frac{1}{C_{2}}}
\end{gathered}
$$

Then we come to Eqs.(32) and (33). 